\title{
VLT/UVES spectroscopy of V4332 Sagittarii in 2005: The best view on a decade-old stellar-merger remnant ${ }^{\star}, \star \star$
}

\author{
R. Tylenda ${ }^{1}$, S. K. Górny ${ }^{1}$, T. Kamiński ${ }^{2}$, and M. Schmidt ${ }^{1}$
}

\author{
1 Department of Astrophysics, Nicolaus Copernicus Astronomical Center, Rabiańska 8, 87-100 Toruń, Poland \\ e-mail: tylenda@ncac.torun.pl \\ 2 European Southern Observatory, Alonso de Córdova 3107, Vitacura, Santiago, Chile
}

Received 28 December 2014 / Accepted 22 March 2015

\section{ABSTRACT}

\begin{abstract}
Context. The source V4332 Sgr is a red transient (red nova) whose eruption was observed in 1994. The remnant of the eruption shows a unique optical spectrum: strong emission lines of atoms and molecules superimposed on an M-type stellar spectrum. The stellar-like remnant is not directly observable, however. It is presumably embedded in a disc-like dusty envelope seen almost face-on. The observed optical spectrum is assumed to result from interactions of the central-star radiation with dust and gas in the disc and outflows initiated in 1994.

Aims. We aim at studying the optical spectrum of the object in great detail to better understand the origin of the spectrum and the nature of the object.

Methods. We reduced and measured a high-resolution $(R \simeq 40000)$ spectrum of V4332 Sgr obtained with VLT/UVES in April/May 2005. The spectrum comes from the ESO archives and is the best quality spectrum of the object ever obtained.

Results. We identified and measured over 200 emission features belonging to 11 elements and 6 molecules. The continuous, stellarlike component can be classified as $\sim \mathrm{M} 3$. The radial velocity of the object is $\sim-75 \mathrm{~km} \mathrm{~s}^{-1}$ as derived from narrow atomic emission lines. The interstellar reddening was estimated to be $0.35 \leq E_{B-V} \leq 0.75$. From radial velocities of interstellar absorption features in the $\mathrm{Na}$ I D lines, we estimated a lower limit of $\sim 5.5 \mathrm{kpc}$ to the distance of V4332 Sgr. When compared to spectroscopic observations obtained in 2009, the spectrum of V4332 Sgr considerably evolved between 2005 and 2009. The object significantly faded in the optical (by $\sim 2$ mag in the $V$ band), which resulted from cooling of the main remnant by 300-350 K, corresponding to its spectral-type change from M3 to M5-6. The object increased in luminosity by 50\%, however, implying a significant expansion of its dimensions. Most of the emission features seen in 2005 significantly faded or even disappeared from the spectrum of V4332 Sgr in 2009. These resulted from fading of the optical central-star radiation and a decrease of the optical thickness of the cirumstellar matter, presumably due to its expansion. V4332 Sgr bears several resemblances to V1309 Sco, which erupted in 2008. This can indicate a similar nature of the eruptions of the two objects. The outburst resulted from merger of a contact binary in V1309 Sco.
\end{abstract}

Key words. stars: activity - circumstellar matter - stars: emission-line, Be - stars: individual: V4332 Sgr - stars: late-type stars: mass-loss

\section{Introduction}

The source V4332 Sagittarii (V4332 Sgr) was discovered as Nova Sgr 1994 in February 1994 (Hayashi et al. 1994). Spectroscopic observations showed narrow Balmer lines in emision superimposed on a K-type (super)giant spectrum, however, that quickly evolved to $\mathrm{M}$ type (super)giant (Tomaney et al. 1994; Martini et al. 1999). This spectral apearance and evolution was at variance with what is observed in classical novae, but bore a resemblance to the luminous red variable observed in M31 in 1988 (M31 RV; Mould et al. 1990). At present, stellar eruptions of this type are called red transients, intermediate-luminosity red transients, red novae, luminous red novae, or V838 Mon-type objects. The latter name comes from the gigantic eruption of V838 Monocerotis observed in 2002 (Munari et al. 2002; Crause et al. 2003), which elicited great interest in astrophysicists, as

\footnotetext{
* Tables 2-4 are available in electronic form at http: //www . aanda.org

$\star \star$ The reduced spectrum (as a FITS file) is only available at the CDS via anonymous ftp to cdsarc.u-strasbg. fr $(130.79 .128 .5)$ or via http://cdsarc.u-strasbg.fr/viz-bin/qcat?]/A+A/578/A75
}

well as public media, partly due to the spectacular light-echo event accompanying the outburst (Bond et al. 2003).

In addition to these three objects, the class of red transients in our Galaxy includes V1309 Scorpii (V1309 Sco; Mason et al. 2010) and OGLE-2002-BLG-360 (Tylenda et al. 2013). V1148 Sagittarii (Nova Sgr 1943) probably also belonged to this class, as can be inferred from its spectral evolution described by Mayall (1949). There is also growing observational evidence that CK Vulpeculae (CK Vul, Nova Vul 1670; Shara et al. 1985) was a red transient and not a classical nova (Kato 2003; Tylenda et al. 2013; Kamiński et al. 2015a). A few extragalactic objects, usually referred to as intermediate-luminosity optical transients, for instance, M85 OT2006 (Kulkarni et al. 2007), NGC300 OT2008 (Bond et al. 2009; Berger et al. 2009), and SN 2008S (Smith et al. 2009), might be of a similar nature.

Although they are different in light curve, time scale, and peak luminosity, red transients always show a similar spectral evolution: in course of the eruption, the objects evolve to progressively lower effective temperatures and decline as M-type (super)giants. Their remnants also resemble a late M-type (super)giants with a significant (often dominating) infrared excess. 
Several mechanisms have been proposed to explain the redtransient events, including an unusual nova (Iben \& Tutukov 1992), a late He-shell flash (Lawlor 2005), and a stellar merger (Soker \& Tylenda 2003). They have been critically discussed in Tylenda \& Soker (2006). These authors concluded that the only mechanism that can satisfactorily account for the observational data of red transients is a merger of two stars. For the case of V838 Mon they argued that this eruption might have been due to a merger of a low-mass pre-main-sequence star with an $\sim 8 M_{\odot}$ main-sequence star.

The source V1309 Sco, which erupted in 2008 (Mason et al. 2010), appeared to be a sort of Rosetta stone for understanding the nature of red transients. Thanks to the archive data from the Optical Gravitational Lensing Experiment (OGLE; Udalski 2003), it was possible to follow the photometric evolution of the object during six years before the outburst (Tylenda et al. 2011). The result was amazing: the progenitor of V1309 Sco was a contact binary that quickly lost its orbital angular momentum and evolved into a merger of the components. Thus V1309 Sco provided strong evidence that the red transients are indeed caused by stellar mergers.

After the 1994 outburst, V4332 Sgr was almost forgotten by astrophysicists and observers. No observations of the object were made until 2002, when V838 Mon erupted and astronomers realized that these two objects most probably belong to the same class. Then V4332 Sgr regained astrophysical interest. Several spectroscopic observations made in 2002-2003 revealed an unusual optical spectrum for stellar objects: strong and numerous emission lines of atoms and molecules were superimposed on a weak, early-M-type stellar spectrum (Banerjee \& Ashok 2004; Tylenda et al. 2005; Kimeswenger 2006). In addition, strong bands of $\mathrm{AlO}$ in emission were detected in the near-IR spectral region (Banerjee et al. 2003).

A detailed study of the emission-line spectrum and the spectral energy distribution (SED) of V4332 Sgr led Kamiński et al. (2010) to conclude that the main remnant of the 1994 eruption is now obscured to us, most probably the central object is embedded in a dusty disc seen almost edge-on. The observed optical spectrum is assumed to be produced by interactions of the central star's radiation with the matter in the disc and the outflows originating from the 1994 eruption: the M-type continuum results from scattering on dust grains, while the emission-line spectrum is due to resonant scattering by atoms and molecules. This conclusion was subsequently confirmed by polarimetric (Kamiński \& Tylenda 2011) and spectropolarimetric (Kamiński \& Tylenda 2013) observations, which showed that the optical continuum is strongly polarized, while the emission features are mostly unpolarized.

We present an optical spectrum of V4332 Sgr obtained in 2005. The data come from the archives of the Very Large Telescope (VLT) and were not published before. The quality and resolution of the spectrum is exceptional; the data present the best quality spectrum of V4332 Sgr ever obtained. Since 2005, the object has significantly faded (see Fig. 1) and its spectrum has considerably evolved (see e.g. Barsukova et al. 2014). Therefore the spectrum described and analysed in the next sections presents a unique set of data on V4332 Sgr, which was the main reason for us to reduce and publish the data.

\section{Observations and data reduction}

We have found high resolution spectra of V4332 Sgr in the ESO data archives. They have been carried out with UVES/VLT in

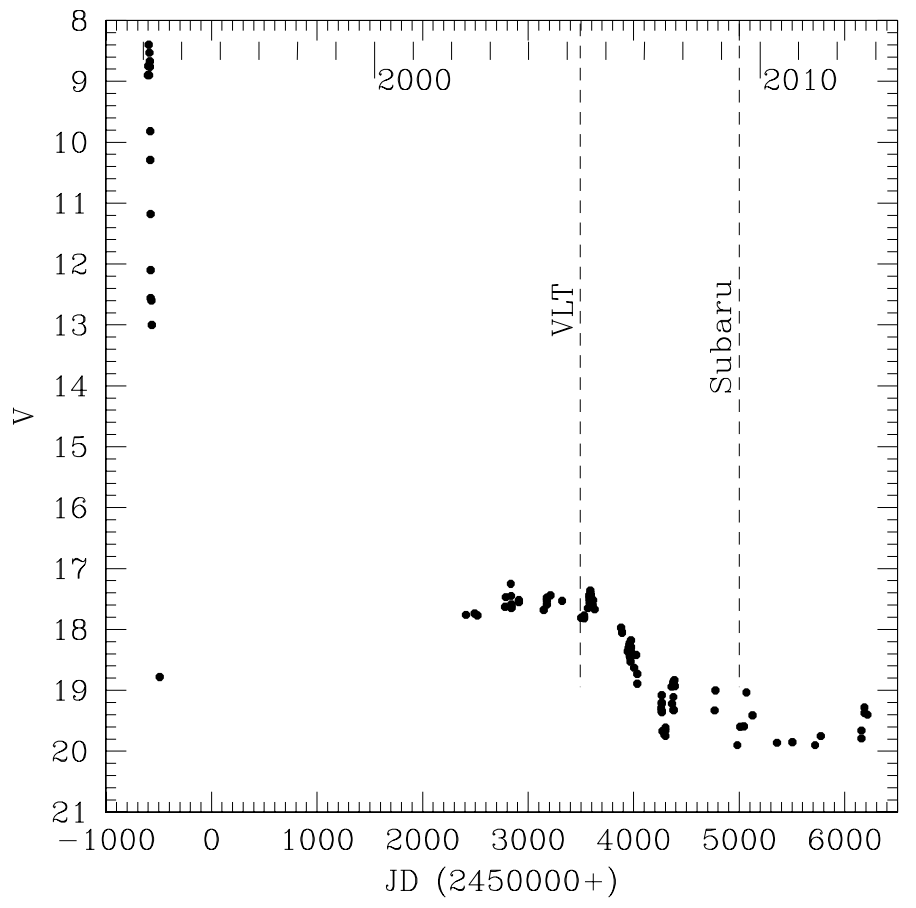

Fig. 1. Light curve of V4332 Sgr in the $V$ band. Vertical dashed lines indicate the time moments of the VLT spectroscopy we analysed and the observations obtained with the Subaru telescope in June 2009 that are described in Kamiński et al. (2010).

2005 on 22 April and 12 May. The observations were made in the framework of the 075.D-0511 (PI: Banerjee) observing programme. The spectra were obtained with three different spectrograph settings covering the range $3756-10253 \AA$ with two gaps at 5750-5833 $\AA$ and 8520-8656 $\AA$. The technical details of the observations are provided in Table 1.

Figure 1 shows the light curve of V4332 Sgr in the $V$ photometric band since the discovery of the object in February 1994. The data are taken from the compilation of V. Goranskij ${ }^{1}$ (see Barsukova et al. 2014, for the light curves in different photometric bands). Two vertical dashed lines indicate the time moments of the VLT spectroscopic observations we analysed and the observations obtained with the Subaru telescope in June 2009 that were presented in Kamiński et al. (2010).

One blue and two different red standard UVES configurations were used to observe the target. The observations centred on $8600 \AA$ initially split into two exposures were later merged. Since in the case of the red configurations there are two separate lower and upper parts, the final spectra consist of five independently registered elements. The CCDs were read with $2 \times 2$ binning in all configurations. The total useful spectral range covers 3760-9500 ^, with two small overlapping regions of individual sections and some gaps between lower and upper parts of the red configurations.

From the ESO data archive we have downloaded the spectra in their raw, unreduced form together with the full set of reduction and calibration files for each of the standard configurations. The reduction and calibration of the spectra was made with the ESO-MIDAS reduction software.

The background was subtracted from flat-field, arc, and science frames. The Th-Ar lamps were used for wavelength

1 http://jet.sao.ru/ goray/v4332sgr.ne3 

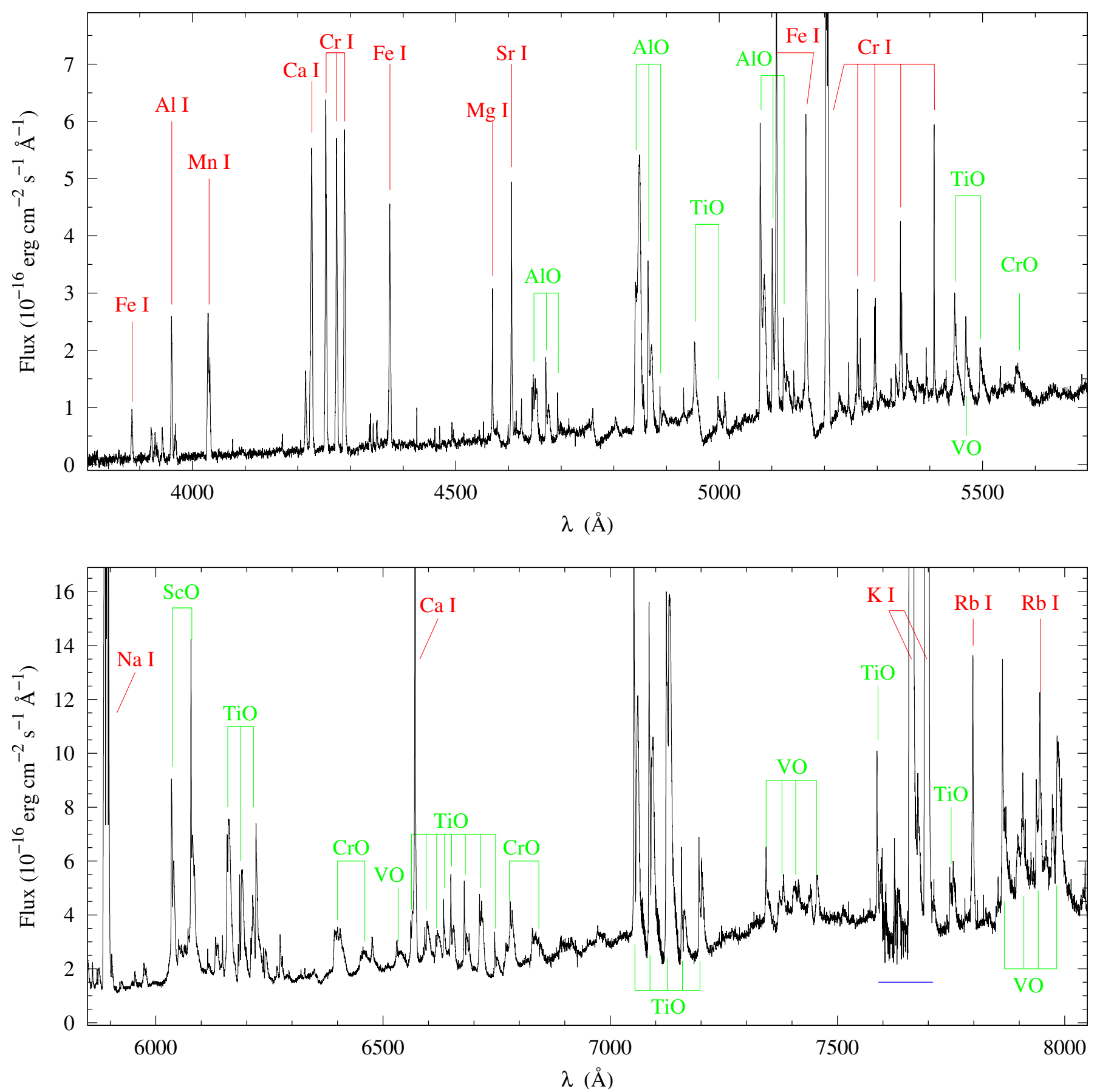

Fig. 2. Spectrum of V4332 Sgr obtained in April-May 2005 with the UVES/VLT. The displayed spectrum was smoothed from the original resolution with boxcar 10. The strongest atomic (red) and molecular (green) spectral features are indicated. A blue horizontal bar indicates the spectral region affected by telluric absorption bands.

calibration. Flat-fielding was performed in the pixel-pixel space. The signs of cosmic rays and other defects of the CCD were removed from each science frame using the standard MIDAS procedures. The extracted spectra were wavelength calibrated and corrected for atmospheric extinction. The flux calibration was performed using the master response calibration files prepared by ESO for each of the standard UVES configurations. Telluric lines were partially removed using Molecfit (Smette et al. 2015). The procedure left residuals below $20 \%$ of the original strength of the telluric lines. The individual heliocentric velocity corrections were applied to each individual spectrum of V4332 Sgr. As a last step, the five parts were combined into a single file, averaging the overlapping regions.

\section{Spectrum}

The final flux-calibrated 1D spectrum of V4332 Sgr is presented in Fig. 2. The spectrum above $\sim 8100 \AA$ is not shown in the figure because it is heavily disturbed by telluric absorption lines and only shows a few emission features of the object (see Table 3 ). The wavelength is given in the heliocentric rest frame, and the flux is in units of $10^{-16} \mathrm{erg} \mathrm{s}^{-1} \mathrm{~cm}^{-2} \AA^{-1}$. The strongest atomic and molecular features in emission are indicated in the figure.

The atomic line identification was based mainly on the NIST Atomic Spectra Database (Kramida et al. 2013) ${ }^{2}$, the Atomic

2 http://physics.nist.gov/asd 
Table 1. Log of observations of V4332 Sgr with UVES/VLT.

\begin{tabular}{clccc}
\hline \hline Date and time & Configuration & Range $(\AA)$ & Resolution & Exp. time \\
\hline 2005.04.22 06:22:01 & RED 580 & $4727-6835$ & 42310 & $3000 \mathrm{~s}$ \\
2005.05.12 06:10:19 & RED 860 & $6650-10250$ & 42310 & $1480 \mathrm{~s}$ \\
2005.05.12 06:10:23 & BLUE 437 & $3730-5000$ & 40970 & $2960 \mathrm{~s}$ \\
2005.05.12 06:43:16 & RED 860 & $6650-10250$ & 42310 & $1100 \mathrm{~s}$ \\
\hline
\end{tabular}

Line List v2.04 by van Hoof $^{3}$, and on the multiplet tables by Moore (1945). All the identified atomic lines seen in emission in the spectrum of V4332 Sgr are listed in Table 2. The observed wavelengths (in $\AA$ ) of the lines are given in Col. (1) of the table, while their laboratory wavelengths and ion identification can be found in Cols. (2) and (3), respectively. Column (4) presents the measured fluxes (in units of $10^{-16} \mathrm{erg} \mathrm{s}^{-1} \mathrm{~cm}^{-2}$ ) of the lines with their estimated uncertainties. Column (5) gives the full widths at half maximum (FWHM in $\AA$ ) of the lines. The last column lists notes on the lines and their measurements. The explanations of the symbols used in Table 2 are given in Table 4.

All the identified molecular bands seen in emission in the spectrum of V4332 Sgr are listed in Table 3. The method used to identify the bands with appropriate references for molecular data can be found in Kamiński et al. (2009) and Kamiński et al. (2010). The first column of the table gives wavelengths of the bands. These are mostly values from laboratory measurements and refer to the heads of the bands. In a few cases, the wavelengths are results of theoretical estimates. The identification of the bands is provided in Col. (2), while Col. (3) displays the measured fluxes (in $10^{-16} \mathrm{erg} \mathrm{s}^{-1} \mathrm{~cm}^{-2}$ ) in the bands with their accuracies. The last column gives notes on the bands and their measurements. The symbols used in these two columns are explained in Table 4.

\section{Spectral classification of the stellar-like continuum}

To estimate the spectral type of the stellar-like continuum observed in V4332 Sgr, we attempted to fit standard and model spectra to the results of our observations. Standard stellar spectra were taken from Jacoby et al. (1984), while model atmosphere spectra were obtained using the MARCS grid (Gustafsson et al. 2008).

Figure 3 shows M2 and M4 giant spectra fitted to the observations. An interstellar reddening was applied to the standard spectra to obtain a good fit at the short- and long-wavelength edges of the spectra. These were $E_{B-V}=0.4$ for the M2 and 0.15 for the M4 spectrum. As can be seen from Fig. 3, neither of the two standard spectra satisfactorily fits the observations. The M2 spectrum presents absorption bands somewhat too shallow when compared to the observed continuum, while its flux in the middle spectral region, that is, between $\sim 5000 \AA$ and $\sim 6500 \AA$, is systematically too high. In contrast, the absorption bands of the M4 spectrum are too deep, and its flux in the middle region is too low. The observed continuum generally lies in between the M2 and M4 standard spectra, suggesting that an M3 giant spectral type probably reproduce the observations adequately.

Indeed, as shown in Fig. 4, the M3 standard spectrum, as well as the MARCS model spectrum calculated for stellar parameters typical for an M3 (super)giant $\left(T_{\text {eff }}=3600 \mathrm{~K}, \log g=\right.$ 0.5, Levesque et al. 2005), fit the observed continuum. Some

\footnotetext{
3 http://www.pa.uky.edu/ peter/atomic/
}

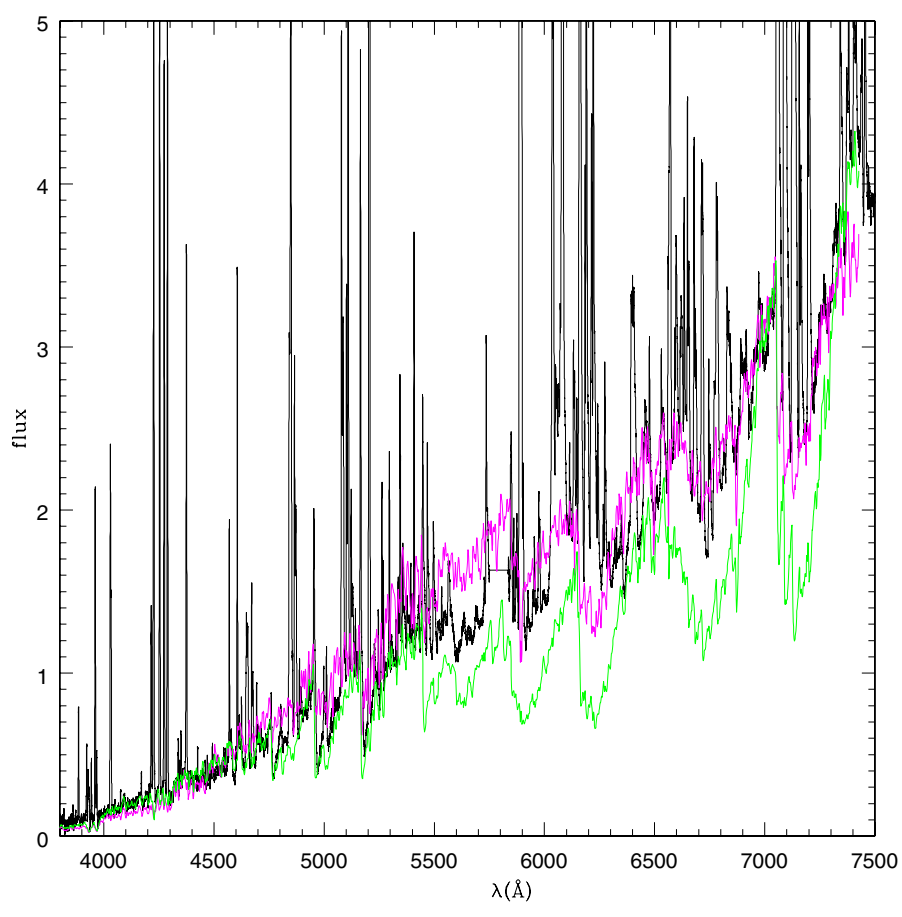

Fig. 3. Standard giant spectra fitted to the observed spectrum of V4332 Sgr (black). Magenta: M2 standard spectrum reddened with $E_{B-V}=0.4$. Green: M4 standard spectrum reddened with $E_{B-V}=0.15$.

absorption bands, for example that at $\sim 6200 \AA$, may seem to be too deep in the reference spectra compared to the observed band. However, in the spectrum of V4332 Sgr these absorption features are partly filled by strong, partly overlapping emissions. Note that the two reference spectra shown in Fig. 4 were reddened with $E_{B-V}=0.35$.

\section{Interstellar reddening}

The interstellar extinction towards V4332 Sgr was estimated in a number of papers (Martini et al. 1999; Tylenda et al. 2005; Kimeswenger 2006). These results as well as their own determinations were discussed in Kamiński et al. (2010). Most of the estimates relied on a comparison of the observed continuum of V4332 Sgr with stellar standards. Kamiński et al. (2010) finally adopted $E_{B-V}=0.32$, although the values varied between 0.22 and 0.45 . This value agrees well with our analysis in Sect. 4, where the best fit to the observed continuum was obtained with an $\mathrm{M} 3$ giant spectrum reddened with $E_{B-V} \simeq 0.35$.

However, as proposed in Kamiński et al. (2010) and confirmed by spectropolarimetric observations in Kamiński \& Tylenda (2013), the observed stellar continuum in V4332 Sgr is not a direct spectrum of the main, central object, but results from scattering the central-star radiation on dust grains. Since scattering on cosmic dust grains usually causes the incident spectrum to be bluer, the result of $E_{B-V}=0.35$ obtained in Sect. 4, as well 


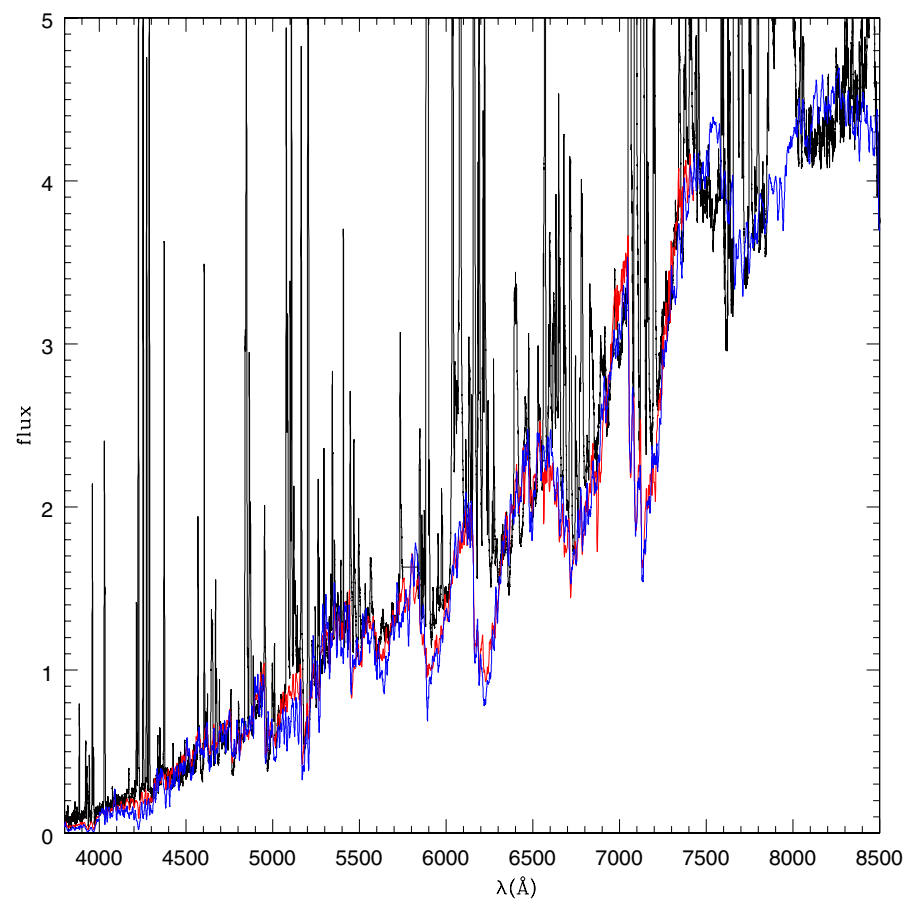

Fig. 4. Standard and model giant spectra fitted to the observed spectrum of V4332 Sgr (black). Red: M3 standard spectrum reddened with $E_{B-V}=0.35$. Blue: model atmosphere $\left(T_{\text {eff }}=3600 \mathrm{~K}, \log g=0.5\right)$ spectrum reddened with $E_{B-V}=0.35$.

as that in Kamiński et al. (2010), may be regarded as a lower limit to the reddening of V4332 Sgr.

Kamiński et al. (2010) proposed that the emission features observed in the spectrum of V4332 Sgr result from resonant scattering of the central-star radiation by molecules and atoms in a circumstellar matter. They also concluded that the strongest emission lines, such as Na I 5890, $5896 \AA$ and K I 7665, $7699 \AA$, are optically thick in the sense that they scatter all the incident stellar radiation. The main argument was that the observed intensity ratio of the two components of the $\mathrm{NaI}$ and K I doublets was close to $1: 1$, while in the optically thin case this ratio should be $2: 1$. The spectrum of V4332 Sgr we discuss here shows much more numerous and strong emission features than that of Kamiński et al. (2010). In addition to the Na I and K I doublets, we therefore searched for other emission lines, which are likely to be optically thick. These are the Cr I lines at 4254, 4275, $4290 \AA$. In the optically thin case, the intensity ratio of these lines should be 1.8:1.4:1.0. As can be seen from Table 2, the observed ratio is 1.02:0.89:1.0. Almost certainly, the Ca I $4227 \AA$ line is also optically thick because it presents the highest value of the product of element abundance and transition probability of all the observed emission features in our spectrum. Finally, the line ratio of the Mn I lines at 4031, 4033, $4034 \AA$ suggests that these lines are almost optically thick, at least the first one. The theoretical optically thin line ratio for these Mn I lines is 2.0:1.5:1.0. The lines are strongly blended, but from the observed profile we can estimate a ratio of 1.4:0.8:1.0.

In this way, we have a set of emission lines whose monochromatic fluxes in the central, most opaque regions of their profiles are expected to measure the monochromatic flux from the central star. These lines span a wide spectral region, 4000-8000 A, which means that they can be used to estimate the interstellar reddening when compared to a reference spectrum that is assumed to represent the spectrum of the central object of

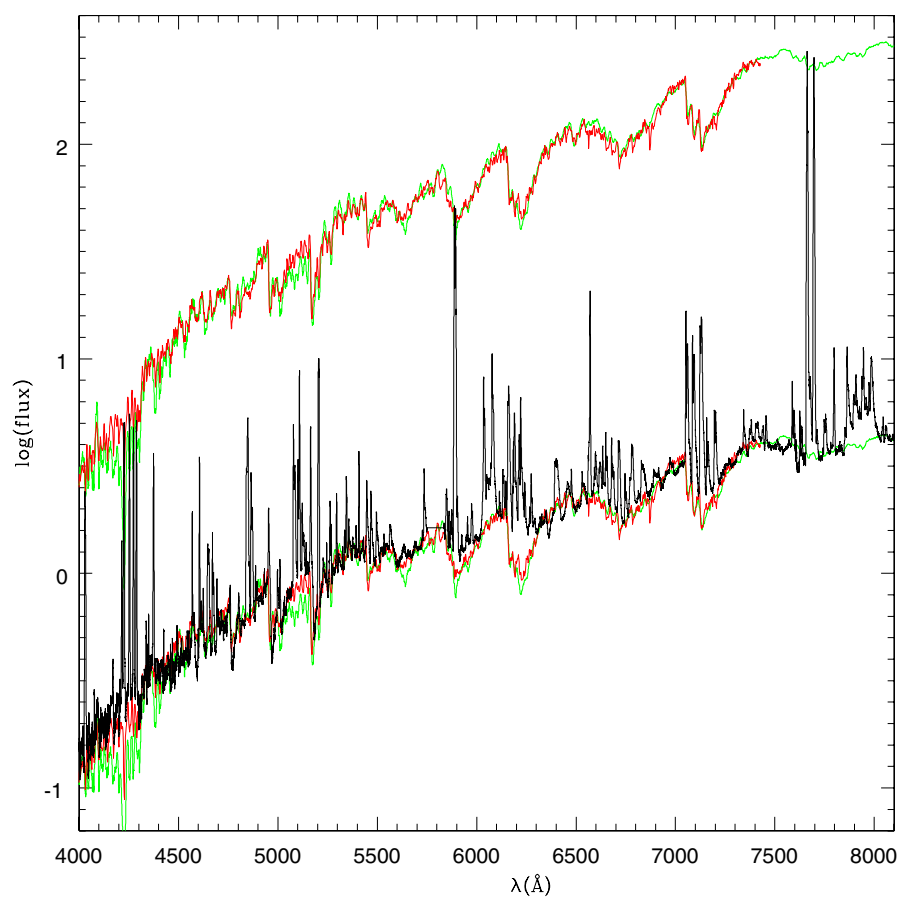

Fig. 5. Reference (standard and model giant) spectra compared to the observed spectrum of V4332 Sgr (black). Red: M3 standard spectrum, green: model atmosphere $\left(T_{\mathrm{eff}}=3600 \mathrm{~K}, \log g=0.5\right)$. Reference spectra in the lower plots are reddened with $E_{B-V}=0.35$. Reference spectra in the upper plots are reddened with $E_{B-V}=0.75$ and shifted upward to match the highest fluxes of the strongest emission lines.

V4332 Sgr. This is done in Fig. 5. The lower part of the figure presents essentially the same as Fig. 4 (i.e. the standard M3 and model, $T_{\text {eff }}=3600 \mathrm{~K}$, spectra reddened with $E_{B-V}=0.35$ ( $R \equiv A_{V} / E_{B-V}=3.1$ was used throughout this paper) and compared to the observed spectrum) but in the logarithm scale of the flux. The upper plots show the same reference spectra, but shifted upwards to match the highest fluxes of the strongest, that is, optically thick, emission lines, namely MnI $4031 \AA$, Ca I $4227 \AA$, Cr I 4254, 4275, $4290 \AA$, Na I 5890, $5896 \AA$, and KI 7665, $7699 \AA$. A pure upward shift did not suffice to fit the level of the highest fluxes in the lines. It was also necessary to increase the reddening to $E_{B-V} \simeq 0.75$. Thus we can conclude that the emission-line spectrum of V4332 Sgr is significantly more reddened than the stellar-like continuum. As discussed above, the difference in the reddening is most likely due to the bluering of the stellar continuum that is scattered on circumstellar dust grains.

There is a process, however, that can affect the relative fluxes of the lines we have used to estimate the reddening of the emission spectrum. All the lines we have considered above are from resonant transitions in atoms. If the lines are optically thick, the resonance photons must suffer from numerous scattering before they escape the emitting region. This increases the chances of being absorbed by dust in the region. Since the absorption coefficent of dust grains is expected to increase with decreasing wavelength, the lines at 4000-4300 would suffer more from dust absorption than the lines at 7600-7800 ̊. Detailed line transfer calculations are neccesary to investigate this effect, which is beyond the scope of the present study. The process, if effective, would mimic an additional interstellar extinction. Therefore we conclude from the discussion in this section that the interstellar reddening to V4332 is $0.35 \lesssim E_{B-V} \lesssim 0.75$. 
Table 5. Components of the interstellar absorption in the profile of the Na I $5890 \AA$ Å line (see Fig. 6).

\begin{tabular}{cccc}
\hline \hline Designation & $\lambda_{\mathrm{obs}}(\AA)$ & $V_{\mathrm{r}}\left(\mathrm{km} \mathrm{s}^{-1}\right)$ & Distance $(\mathrm{kpc})$ \\
\hline $\mathrm{a}$ & 5889.779 & -8.7 & 0.48 \\
$\mathrm{~b}$ & 5890.104 & 7.8 & 2.50 \\
$\mathrm{c}$ & 5890.573 & 31.7 & 4.23 \\
$\mathrm{~d}$ & 5890.817 & 44.2 & 4.85 \\
$\mathrm{e}$ & 5891.110 & 59.1 & 5.44 \\
\hline
\end{tabular}

\section{Radial velocity}

All clear absorption features in the spectrum of V4332 Sgr can be identified as molecular bands (mostly TiO). They are wide, shallow, and usually filled near their heads with strong emission components. We were not able to identify any atomic absorption line. This precludes a reliable determination of the radial velocity of V4332 Sgr on the basis of its absorption spectrum.

However, many of the observed atomic emission lines are relatively narrow ( $F W H M \lesssim 1 \AA$, see Col. 5 in Table 2$)$, and they can be used to determine the radial velocity of the object. The strongest emission lines are not particularly suitable for this purpose because they are wide and their observed wavelengths are poorly determined. We have selected a set of 28 unblended lines of medium intensities. All of them gave consistent values of the radial velocity, ranging between -83 and $-68 \mathrm{~km} \mathrm{~s}^{-1}$. The mean value and standard deviation derived from this sample are $-75.1 \pm 3.1 \mathrm{~km} \mathrm{~s}^{-1}$. This value can be compared with $-65 \pm 7 \mathrm{~km} \mathrm{~s}^{-1}$ obtained in Kamiński et al. (2010), but that was an estimate based on strong and wide emission lines.

We note that these estimates refer to the line-emitting region, and it is not clear to what extent they measure the radial velocity of the main, stellar-like object of V4332 Sgr. If these values measure the real radial velocity of V4332 Sgr, then the object does not follow the standard rotation of the Galaxy (see e.g. Brand \& Blitz 1993) because the heliocentric radial velocity at any distance is expected to be $z-10 \mathrm{~km} \mathrm{~s}^{-1}$ for the position of V4332 Sgr.

\section{Interstellar lines and distance to V4332 Sgr}

There is no reliable estimate of the distance to V4332 Sgr. Martini et al. (1999) proposed 300 pc assuming that the object was a K-type giant at maximum of the 1994 outburst. Tylenda et al. (2005) obtained $1.8 \mathrm{kpc}$ assuming that the progenitor was a solar-type main-sequence star. Kimeswenger (2006) estimated 2.9 or $10 \mathrm{kpc}$ depending on whether luminosity class V or III was assumed for the progenitor. Kamiński et al. (2010) derived a kinematic distance $\gtrsim 1.0 \mathrm{kpc}$ from the radial velocity of the interstellar absorption lines of the Na I 5890, $5896 \AA$, and K I 7665, 7699 $\AA$ lines. These authors identified a single component of these lines in their spectrum of V4332 Sgr. Our spectrum is of a much better quality than that of Kamiński et al. (2010), and we can identify several components of the interstellar Na I lines.

Figure 6 displays the observed profiles of the Na I 5890 and $5896 \AA$ lines superimposed on each other, plotted on the radial velocity scale. Up to five components of the interstellar absorptions are clearly seen, particularly in the Na I $5890 \AA$ line. They are marked with letters in the figure and are listed in Table 5. The observed wavelengths of the individual components were obtained by fitting Gaussian profiles to the observed features. Note that the absorption feature seen at $V_{\mathrm{r}} \simeq 110 \mathrm{~km} \mathrm{~s}^{-1}$ in the

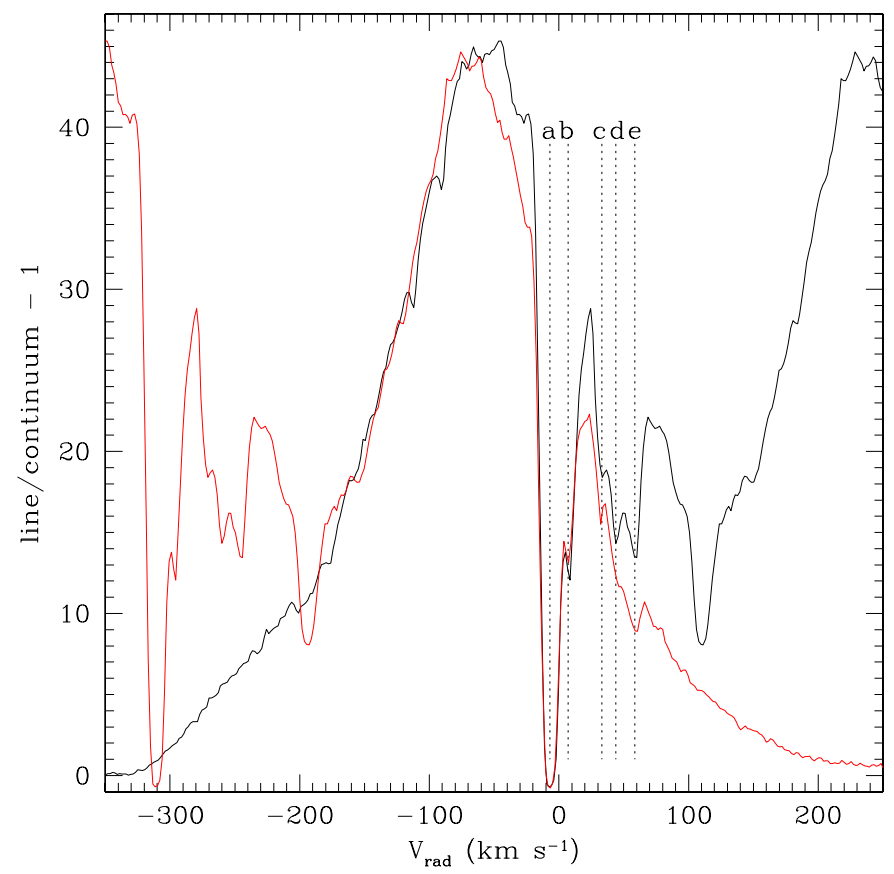

Fig. 6. Profiles of the Na I $5890 \AA$ (black) and $5896 \AA$ (red) lines superimposed on the radial velocity scale. Components of the interstellar absorptions are marked with vertical dotted lines.

Na I $5890 \AA$ line, or at $V_{\mathrm{r}} \simeq-195 \mathrm{~km} \mathrm{~s}^{-1}$ in the Na I $5896 \AA$ line, cannot be of interstellar origin because it has no counterpart in the other line.

The observed wavelengths of the interstellar components given in the second column of Table 5 can be used to calculate heliocentric radial velocities, which are listed in the third column of the table. These values, after being transformed into radial velocities in the LSR frame (adding $12.0 \mathrm{~km} \mathrm{~s}^{-1}$ for the position of V4332 Sgr) and adopting the Galactic rotation curve of Brand \& Blitz (1993), give estimates of distances of the interstellar regions that are responsible for the observed features. The results are listed in the last column of Table 5. From these data we can conclude that V4332 Sgr is located at a distance $\gtrsim 5.5 \mathrm{kpc}$. This together with the Galactic latitude of the object ( $b=-9^{\circ} .40$ ) implies that V4332 Sgr is situated $\gtrsim 0.89$ kpc below the Galactic plane.

\section{Evolution of the spectrum of V4332 Sgr between 2005 and 2009}

Four years after the spectrum described in this paper had been registered, a high-resolution spectrum of V4332 Sgr was obtained by Kamiński et al. (2010) using the Subaru telescope. As can be seen from Fig. 1, the object in meantime faded by $\sim 2 \mathrm{mag}$. in the $V$ band. Figure 7 directly compares the spectra obtained in these two epochs. Two MARCS model spectra fitted to the observed spectra are also plotted in the figure. Note that both model spectra were reddened with $E_{B-V}=0.35$. Note also that the model fit to the 2009 spectrum is not perfect in the long-wavelength range. This point, a possible source of extra absorption in the 7300-7500 $\AA$ range in particular, was discussed in Kamiński et al. (2010).

One of the clear differences between the 2005 and 2009 spectra displayed in Fig. 7 is that the 2009 stellar-like continuum is definitely of a later spectral type than that of 2005. Indeed, Kamiński et al. (2010) classified the 2009 spectrum as M5-6, while we have concluded that it is an M3 type in Sect. 4. This 


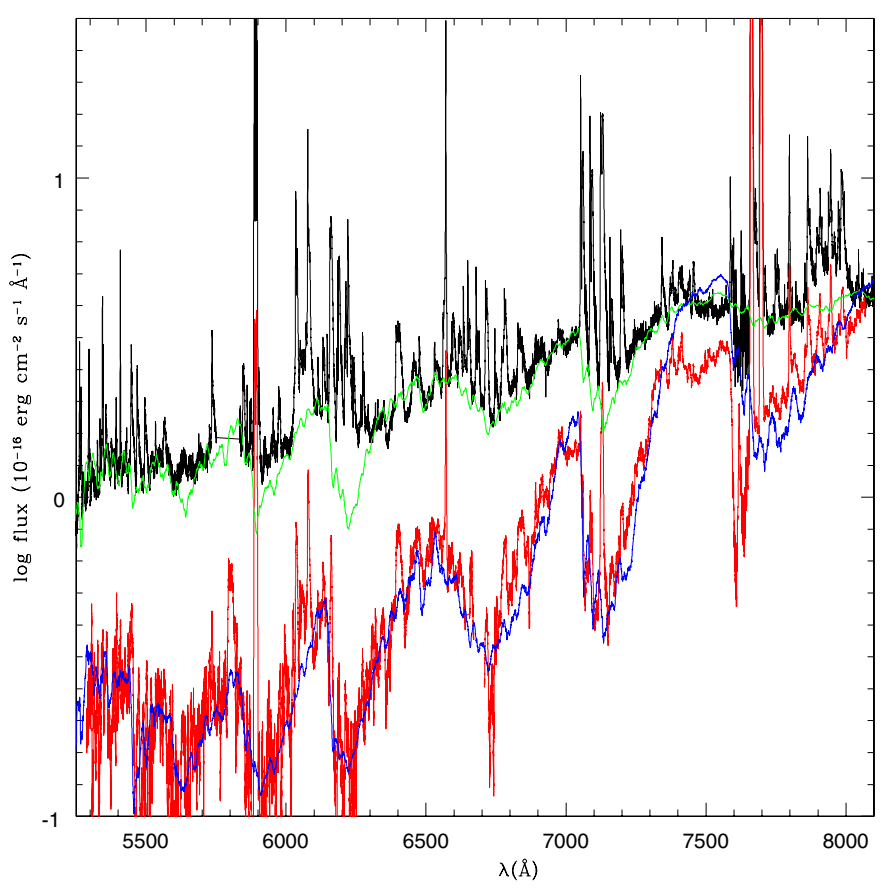

Fig. 7. Comparison of the spectrum of V4332 Sgr in 2005 (black: this paper) to that observed in 2009 (red: Kamiński et al. 2010). Green: model atmosphere $\left(T_{\text {eff }}=3600 \mathrm{~K}, \log g=0.5\right)$ reddened with $E_{B-V}=0.35$ and fitted to the 2009 spectrum. Blue: model atmosphere $\left(T_{\text {eff }}=3300 \mathrm{~K}, \log g=0.0\right)$ reddened with $E_{B-V}=0.35$ and fitted to the 2005 spectrum.

has obvious consequences on the effective temperature of the central stellar-like object in V4332 Sgr. Our MARCS model fitted to the observed spectra has $T_{\text {eff }} \simeq 3300 \mathrm{~K}$ in 2009 compared to $T_{\text {eff }} \simeq 3600 \mathrm{~K}$ in 2005 . However, when fitting the model spectra to the observed spectra in the flux scale, we had to assume that the emitting surface in 2009 increased by a factor of $\sim 2.08$ comparing to 2005 . This implies an increase of the effective radius of the object by a factor of $\sim 1.44$ if a spherically symmetric case is assumed. The luminosity of the object would thus have increased by a factor of $\sim 1.47$ between 2005 and 2009 .

Similar conclusions to these from the optical spectroscopy of V4332 Sgr can be derived from analysing photometric measurements of the object in the optical (BVRI) and near-IR (JHKLM) bands. In Fig. 8 we plot the results of photometric measurements made in 2003 and compiled in Tylenda et al. (2005; red filled symbols), as well as those obtained in 2009 and given in Kamiński et al. (2010; blue open symbols). (There were no nearIR measurements of V4332 Sgr in 2005, but, as can be seen from Fig. 1, the object did not significantly evolve photometrically between 2003 and 2005.) The data were fitted with standard stellar photometric spectra supplemented with black-body dust components in the same way as in Tylenda et al. (2005, for details of the fitting procedure see Tylenda 2005). The final fits (sum of the stellar and dust components reddened with $E_{B-V}=0.35$ ) are shown with the full curves in Fig. 8. The fit for the 2003 data (red in the figure) is the same as those in Tylenda et al. (2005, see their Fig. 5), that is, an M2.7 supergiant $\left(T_{\text {eff }}=3620 \mathrm{~K}\right.$ according to the temperature scale of Levesque et al. 2005) and a $750 \mathrm{~K}$ black-body dust. The fit for 2009 (blue in the figure) consists of an M6.2 $\left(T_{\text {eff }}=3280 \mathrm{~K}\right)$ supergiant and a $900 \mathrm{~K}$ black-body dust. When the luminosities of the components are compared, it appears that both components increased by a similar factor between 2003 and 2009, that is, the stellar component by 1.48 times, the dust component by 1.51 times.

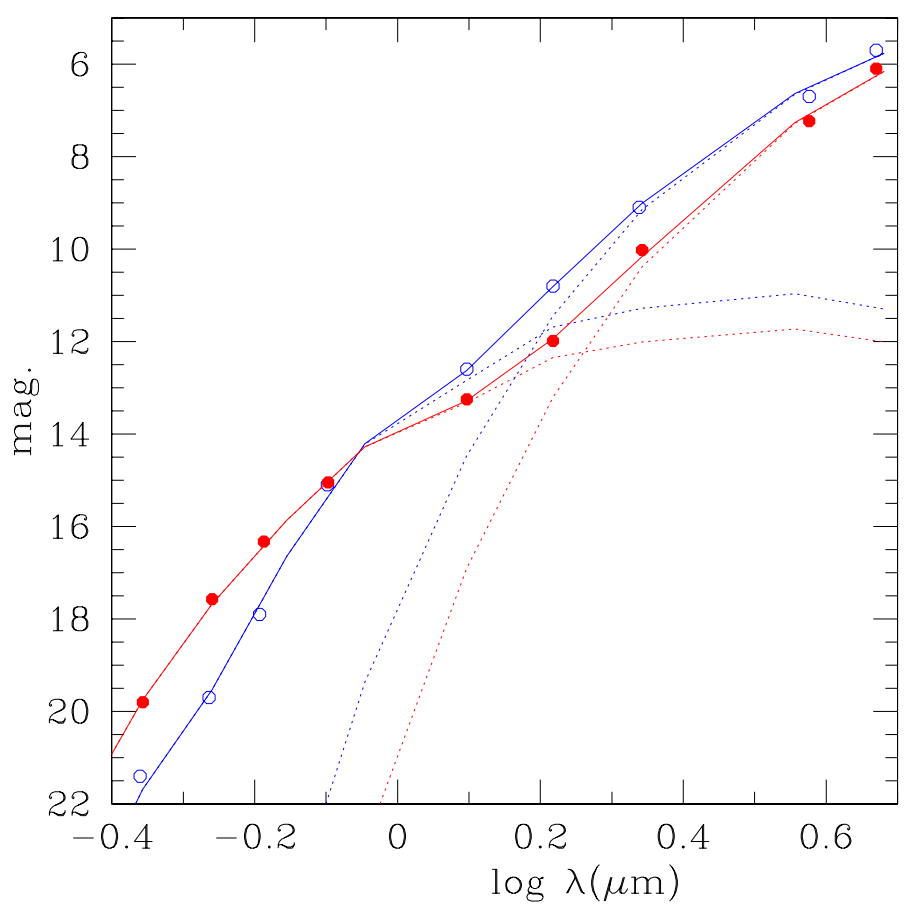

Fig. 8. Comparison of the photometric measurements of V4332 Sgr in 2003 (red filled symbols: Tylenda et al. 2005) to those obtained in 2009 (blue open symbols: Kamiński et al. 2010). Full curves: model photometric spectra obtained by summing a standard stellar component with a black-body dust component (individual components are shown with dotted curves) reddened with $E_{B-V}=0.35$. See text for more details of the modelling.

Thus we can quite safely conclude that the optical decline of V4332 Sgr observed between 2005 and 2007 (see Fig. 1) was due to a decrease of the effective temperature of the central stellar-like object by 300-350 K. The object expanded during this event, however, and its luminosity increased by $~ 50 \%$.

Figure 7 reveals another important difference between the spectra in 2005 and 2009. In the 2009 spectrum the emission features are significantly fainter than in 2005, not only in absolute flux scale, but also relative to the M-type continuum. The fading scale is not the same for all the features, however. The strongest atomic lines, for instance those of $\mathrm{NaI}$ and $\mathrm{KI}$, show a similar strength relative to the local continuum in both epochs. On the other hand, many fainter features that were clearly present in 2005 completely disappeared in 2009. To investigate the problem more quantitatively, we calculated relative contributions of the emission features to the total flux in selected wavelength ranges in both spectra. For a given wavelength range, we derived $F_{\text {obs }}$ as an integral of the observed flux over the wavelength and $F_{\text {mod }}$ as an integral of the MARCS model flux. We assumed that the MARCS spectrum, when fitted to the observed spectrum as in Fig. 7, is a good representation of the stellarlike continuum of V4332 Sgr. Then we can define a parameter $f_{\mathrm{em}}=\left(F_{\mathrm{obs}}-F_{\mathrm{mod}}\right) / F_{\mathrm{obs}}$ as a measure of the relative contribution of the emission features to the total observed flux.

For the whole spectral range common for both spectra, that is, for 5500-8000 $\AA$, we derive $f_{\text {em }}=0.43$ for the 2005 VLT spectrum and 0.25 for the 2009 Subaru spectrum. Thus the global contribution of the emission spectrum to the total observed flux decreased by $\sim 40 \%$ between 2005 and 2009. For narrow spectral ranges encompassing the $\mathrm{NaI}$ and $\mathrm{K} \mathrm{I}$ emission lines, that is, for 5880-5910 $\AA$ and 7650-7720 $\AA$, the result is $f_{\mathrm{em}}=0.93$ and 0.91 in 2005 compared to $f_{\mathrm{em}}=0.91$ and 
0.89 in 2009. Thus, as stated above, the strongest emission features remain practically at the same level when compared to the local continuum. For the 6560-6580 $\AA$ range, which includes the $\mathrm{Ca}$ I line, the figures are $f_{\mathrm{em}}=0.60$ and 0.49 for 2005 and 2009 , which is a decrease of $18 \%$. More important, fading affected molecular emission features. For series of the $\mathrm{TiO} \gamma$ bands observed within 6590-6760 $\AA$ and 7040-7220 $\AA$ we derive $f_{\text {em }}=0.27$ and 0.57 in 2005 , while for the 2009 spectrum the figures are 0.14 and 0.30 . In other words, these emission features faded by a factor of 2 relative to the continuum between 2005 and 2009. Even greater fading affected the VO-B-X(0-0) bands gathered between 7850-8010 $\mathrm{A}$. The result is $f_{\mathrm{em}}=0.37$ and 0.13 (2005 versus 2009) in this case. Thus a general (although not strict) tendency is that the fainter emission feature, the greater the fading effect.

This behaviour can be easily explained within the scenario proposed by Kamiński et al. (2010), according to which the emission features in V4332 Sgr are produced by radiative excitation of atomic and molecular resonant transitions in a circumstellar matter by strong radition from the central stellar-like object, which is invisible to us. The fading of the emission features can then be understood in terms of a decreasing optical thickness of the circumstellar matter, for example due to its expansion. For optically thin transitions the fading would be proportional to the decrease of the optical thickness. However, for optically thick lines, as is probably the case of the $\mathrm{Na}$ I and K I resonant transitions, a modest decrease of their optical thickness would not affect their observed strengths relative to the continuum. The flux in these lines is limited by the available radiative flux from the central source. Thus these emission features fade proportionally to the incident flux, so that their ratio to the observed M-type continum remains unchanged.

\section{Summary and discussion}

We have reduced the best-quality optical spectrum ever obtained for V4332 Sgr. The spectrum was recorded in April/May 2005 with the VLT/UVES equipment and is unique not only because of its quality, but also because the object has considerably evolved since that time. Most of the emission features that were numerous in 2005 disappeared, which means that it will not be possible, in the future, to repeat the observations of the object in a similar stage. Therefore we decided to reduce and publish the spectrum, so that it is made available to the astrophysical community. We presented the spectrum and results of its measurements and general analysis. Forthcoming papers will be devoted to detailed analyses of the emission features, their profiles, intensities, and chemical species that produce them.

The spectrum is dominated by numerous atomic and molecular emission features that are superimposed on an M-type continuous spectrum. Among the emission features we have identified over 70 atomic lines belonging to 11 elements ( $\mathrm{Na}, \mathrm{Mg}$, $\mathrm{Al}, \mathrm{K}, \mathrm{Ca}, \mathrm{Cr}, \mathrm{Mn}, \mathrm{Fe}, \mathrm{Rb}, \mathrm{Sr}$, and $\mathrm{Ba}$; see Table 2) and about 140 bands belonging to 6 molecules (AlO, ScO, TiO, VO, CrO, and YO; see Table 3). There is no other late-type stellar object with an emission spectrum that rich and intense.

The underlying stellar-like continuum in the spectrum of V4332 Sgr can be classified as $\sim$ M3 (see Sect. 4). A giant spectrum of this spectral type fits the observed continuum quite well. Moreover, a MARCS model spectrum calculated for an effective temeprature characteristic of $\sim \mathrm{M} 3$, that is, $\sim 3600 \mathrm{~K}$, reproduced the observations. Strong molecular emissions partly filling the absorption features in the observed continuum did not allow us to analyse the stellar-like continuum of V4332 Sgr in more detail.

From fitting the standard stellar spectrum and the MARCS model spectrum to the observed continuum of V4332 Sgr, we derived an interstellar reddening, $E_{B-V} \simeq 0.35$ (see Sect. 5). This value is consistent with previous determinations (Tylenda et al. 2005; Kimeswenger 2006; Kamiński et al. 2010). However, since the observed continuum is assumed to result from scattering on dust grains, this value probably underestimates the interstellar reddening. Therefore we attempted to estimate the extinction from the strongest emission lines because the highest flux in these lines is expected to follow the flux from the central star. Comparing these data with the standard M3 spectrum and the MARCS model, we obtained $E_{B-V} \simeq 0.75$. This value should be regarded as an upper limit to the real reddening because of possible dust absorption effects of multiply scattered resonantline photons. Thus we concluded that the interstellar reddening of V4332 Sgr is $0.35 \lesssim E_{B-V} \lesssim 0.75$.

In Sect. 6 we derived the radial velocity of V4332 Sgr from a set of relatively narrow medium-intensity emission lines. Our result of $-75.4 \pm 3.5 \mathrm{~km} \mathrm{~s}^{-1}$ is consistent with the results from other determinations (Martini et al. 1999; Tylenda et al. 2005; Kamiński et al. 2010) in the sense that all of them gave negative values, although the values range between -180 to $-56 \mathrm{~km} \mathrm{~s}^{-1}$. As already discussed in Tylenda et al. (2005), these values show that V4332 Sgr does not follow the Galactic rotation curve because from the latter one would expect a heliocentric radial velocity of $z-10 \mathrm{~km} \mathrm{~s}^{-1}$ for any distance for the object coordinates. The problem becomes even more evident if one takes into account the lower limit of the distance derived in Sect. 7, which, assuming the Galactic rotation curve, implies a radial velocity $\gtrsim 60 \mathrm{~km} \mathrm{~s}^{-1}$. One possible explanation is that what we measure in the emission lines is not the radial velocity of the object, but the expansion of the matter ejected in 1994. It is difficult to understand, however, why a decade after the eruption, we would not see the receding part of the ejecta, especially because the radial velocity was determined from medium-intensity lines, which are expected to be optically thin. Another possibility is that V4332 Sgr is not a Galactic disc object, hence its strange radial velocity. This interpretation would be supported by the relatively large distance of the object from the Galactic plane of $\gtrsim 0.89 \mathrm{kpc}$ (see Sect. 7).

Our comparison of the 2005 VLT spectrum to the spectrum obtained in 2009 that was described in Kamiński et al. (2010) (see Sect. 8) showed that the M-type continuum evolved from an $\sim$ M3 spectral type to M5-6, so that the effective temperature of the central stellar object decreased by $300-350 \mathrm{~K}$. The same conclusion also results from photometric measurements made in 2003 and 2009. This relatively small ( 10\%) decrease in the effective temperature is fully responsible for the optical fading of the object observed in 2006. The object expanded during this event, however, so that its luminosity increased by $\sim 50 \%$. This is evident not only from fitting the optical observations, but also from the infrared photometry, which is of particular importance because the infrared emission dominates the observed SED of the object (see e.g. Kamiński et al. 2010). The origin of this behaviour is not clear, but it might be a manifestation of a long-term relaxation of the remnant of the presumable merger event in 1994. The main object is embedded in a massive dusty envelope, probably in the form of a thick disc. An accretion event from the envelope to the central stellar object can be invoked here.

The good resolution and quality of the spectrum we analysed allowed us to detect several components of the interstellar 
absorption in the Na I 5890 and $5896 \AA$ lines (see Sect. 7). The radial velocities of these features, if interpreted as due to interstellar matter moving according to the Galactic rotation curve, imply a lower limit to the distance of V4332 Sgr of $\sim 5.5 \mathrm{kpc}$. This result has important consequences on estimates of the global parameters of V4332 Sgr. In particular, the luminosity of the object during the 1994 eruption, as obtained from photometric measurements in Tylenda et al. (2005), increases now to $\gtrsim 4.5 \times 10^{4} L_{\odot}$, while its effective radius becomes $\gtrsim 450 R_{\odot}$. These values are not as high as those derived for V838 Mon in its 2002 eruption (see e.g. Tylenda 2005), but are of a similar order as those of V1309 Sco in its 2008 eruption (Tylenda et al. 2011).

There are, in fact, other similarities between V4332 Sgr and V1309 Sco. The eruptions of both objects were of a similar time scale, that is, about one month. That of V4332 Sgr was probably slightly longer because its rising part was not observed. The progenitor of V4332 Sgr was variable, and the archive data suggest that it was slowly rising in brightness on a time scale of decades (Kimeswenger 2007; Goranskij et al. 2007). This resembles the slow systematic rise of V1309 Sco observed during a few years before its eruption (Tylenda et al. 2011). The remnants of both objects are strongly dominated by infrared dust emission (Kamiński et al. 2010; Nicholls et al. 2013). Finally, an optical and near-IR spectrum of V1309 Sco obtained in 2012 (Kamiński et al. 2015b) shows an emission-line spectrum similar to that of V4332 Sgr, although not as rich and intense as in the latter case. Clearly, the remnant of V1309 Sco is heavily embedded in dust as was the case of V4332 Sgr. It is therefore tempting to suggest that the nature of the progenitor and the eruption of V4332 Sgr was similar to those of V1309 Sco. We know that the eruption of V1309 Sco resulted from merger of a contact binary (Tylenda et al. 2011).

Acknowledgements. We are grateful to Dipankar P. K. Banerjee, PI of the ESOVLT 075.D-0511 observing programme, for the spectroscopic observations of V4332 Sgr made in 2005, on which this paper is based. Many thanks to the referee for the comments on an earlier version of the paper.

\section{References}

Banerjee, D. P. K., \& Ashok, N. M. 2004, ApJ, 604, L57

Banerjee, D. P. K., Varricatt, W. P., Ashok, N. M., \& Launila, O. 2003, ApJ, 598, L31
Barsukova, E. A., Goranskij, V. P., A. F. Valeev, A. F., \& Zharova, A. V. 2014, Astrophys. Bull., 69, 67

Berger, E., Soderberg, A. M., Chevalier, R. A. et al. 2009, ApJ, 699, 1850

Bond, H. E., Henden, A., Levay, Z. G. et al. 2003, Nature, 422, 405

Bond, H. E., Bedin, L. R., Bonanos, A. Z. et al. 2009, ApJ, 695, L154

Brand, J., \& Blitz, L. 1993, A\&A, 275, 67

Crause, L. A., Lawson, W. A., Kilkenny, D., et al. 2003, MNRAS, 341, 785

Goranskij, V. P., Metlova, N. V., Shugarov, S. Yu., et al. 2007, in The Nature of V838 Mon and its Light Echo, eds. R. L. M. Corradi, \& U. Munari, ASP Conf. Ser., 363, 214

Gustafsson, B., Edvardsson, B., Eriksson, K. et al. 2008, A\&A, 486, 951

Hayashi, S. S., Yamamoto, M., \& Hirosawa, K. 1994, IAU Circ., 5942

Iben, I. Jr., \& Tutukov, A. V. 1992, ApJ, 389, 369

Jacoby, G. H., Hunter, D. A., \& Christian, C. A. 1984, ApJS, 56, 257

Kamiński, T., \& Tylenda, R. 2011, A\&A, 527, A75

Kamiński, T., \& Tylenda, R. 2013, A\&A, 558, A82

Kamiński, T., Schmidt, M., Tylenda, R., Konacki, M., \& Gromadzki, M. 2009, ApJS, 182, 33

Kamiński, T., Schmidt, M., \& Tylenda, R. 2010, A\&A, 522, A75

Kamiński, T., Menten, K. M., Tylenda, R. et al. 2015a, Nature, 520, 322

Kamiński, T., Mason, E., Tylenda, R., \& Schmidt, M. R. 2015b, A\&A, submitted Kato, T. 2003, A\&A, 399, 695

Kimeswenger, S. 2006, Astron. Nachr., 327, 44

Kimeswenger, S. 2007, in The Nature of V838 Mon and its Light Echo, eds. R. L. M. Corradi, \& U.Munari, ASP Conf. Ser., 363, 197

Kramida, A., Ralchenko, Yu., Reader, J., \& NIST ASD Team 2013, NIST Atomic Spectra Database (v. 5.1)

Kulkarni, S. R., Ofek, E. O., Rau, A. et al. 2007, Nature, 447, 458

Lawlor, T. M. 2005, MNRAS, 361, 695

Levesque, E. M., Massey, P., Olsen, K. A. G., et al. 2005, ApJ, 628, 973

Martini, P., Wagner, R. M., Tomaney, A., et al. 1999, AJ, 118, 1034

Mason, E., Diaz, M., Williams, R. E., Preston, G., \& Bensby, T. 2010, A\&A, 516, A108

Mayall, M. W. 1949, AJ, 54, 191

Moore, C. E. 1945, A multiplet table of astrophysical interest, Contributions from the Princeton University Observatory, vol. 20

Mould, J., Cohen, J., Graham, J. R. et al. 1990, ApJ, 353, L35

Munari, U., Henden, A., Kiyota, S., et al. 2002, A\&A, 389, L51

Nicholls, C. P., Melis, C., Soszyński, I., et al. 2013, MNRAS, 431, L33

Shara, M. M., Moffat, A. F. J., \& Webbink, R. F. 1985, ApJ, 294, 271

Smette, A., Sana, H., Noll, S., et al. 2015, A\&A, 576, A77

Smith, N., Ganeshalingam, M., Chornock, R., et al. 2009, ApJ, 697, L49

Soker, N., \& Tylenda, R. 2003, ApJ, 582, L105

Tomaney, A., Rich, R. M., Wagner, R. M., et al. 1994, IAU Circ, 5949

Tylenda, R. 2005, A\&A, 436, 1009

Tylenda, R., \& Soker, N. 2006, A\&A, 451, 223

Tylenda, R., Crause, L. A., Górny, S. K., \& Schmidt, M. R. 2005, A\&A, 439, 651

Tylenda, R., Hajduk, M., Kamiński, T., et al. 2011, A\&A, 528, A114

Tylenda, R., Kamiński, T., Udalski, A., et al. 2013, A\&A, 555, A16

Udalski, A. 2003, Acta Astron., 53, 291 
Table 2. Atomic lines of the spectrum of V4332 Sgr.

\begin{tabular}{|c|c|c|c|c|c|}
\hline$\lambda_{\mathrm{obs}}$ & $\lambda_{\mathrm{lab}}$ & Identification & Flux & $F W H M$ & Notes \\
\hline 3860.47 & 3859.91 & $\mathrm{FeI}$ & $0.37( \pm 0.41)$ & 2.1: & $\mathrm{f}$ \\
\hline 3885.48 & 3886.28 & FeI & $2.12( \pm 0.48)$ & 2.55 & \\
\hline 3922.26 & 3922.91 & FeI & $1.38( \pm 0.35)$ & 2.5: & \\
\hline 3929.29 & 3930.30 & $\mathrm{FeI}$ & $0.89( \pm 0.30)$ & 2.1: & \\
\hline 3932.86 & 3933.66 & CaII & $0.77( \pm 0.49)$ & $3.4:$ & \\
\hline 3943.40 & 3944.01 & AlI & $1.07( \pm 0.33)$ & 2.3: & \\
\hline 3960.79 & 3961.52 & AlI & $6.64( \pm 0.38)$ & 2.70 & \\
\hline 3967.84 & 3968.47 & CaII & $1.73( \pm 0.50)$ & 3.5: & \\
\hline 4029.8 & 4030.75 & MnI & $11.60( \pm 1.08)$ & 5.3: & ך \\
\hline 4031.8 & 4033.06 & MnI & & & B \\
\hline 4033.5 & 4034.48 & MnI & & & ЈB \\
\hline 4076.66 & & $?$ & $0.20( \pm 0.08)$ & 0.6 : & $\mathrm{f}$ \\
\hline 4170.99 & & $?$ & $0.58( \pm 0.32)$ & 2.5: & $\mathrm{f}$ \\
\hline 4205.66 & 4206.70 & $\mathrm{FeI}$ & $0.20( \pm 0.11)$ & 0.9: & $\mathrm{f}$ \\
\hline 4215.16 & 4216.18 & FeI & $4.39( \pm 0.41)$ & 3.17 & \\
\hline 4226.9 & 4226.73 & $\mathrm{CaI}$ & $20.17( \pm 0.72)$ & 3.31 & \\
\hline 4253.58 & 4254.33 & $\mathrm{CrI}$ & $18.43( \pm 0.39)$ & 3.00 & \\
\hline 4274.01 & 4274.80 & $\mathrm{CrI}$ & $16.02( \pm 0.39)$ & 3.06 & \\
\hline 4289.08 & 4289.72 & $\mathrm{CrI}$ & $18.04( \pm 0.41)$ & 3.17 & \\
\hline 4336.46 & 4337.57 & $\mathrm{CrI}$ & $0.28( \pm 0.08)$ & 0.58 & \\
\hline \multirow[t]{2}{*}{4338.44} & 4339.45 & $\mathrm{CrI}$ & $0.62( \pm 0.13)$ & 0.98 & ך \\
\hline & 4339.72 & $\mathrm{CrI}$ & & & 」B \\
\hline 4343.39 & 4344.50 & $\mathrm{CrI}$ & $0.34( \pm 0.09)$ & 0.66 & \\
\hline 4349.92 & 4351.05 & $\mathrm{CrI}$ & $0.39( \pm 0.11)$ & 0.87 & $\mathrm{~b}$ \\
\hline \multirow[t]{2}{*}{4350.79} & 4351.76 & $\mathrm{CrI}$ & $0.30( \pm 0.08)$ & 0.60 & $\mathrm{~b}$ \\
\hline & 4359.63 & CrI & & & $\mathrm{p}$ \\
\hline 4370.22 & 4371.26 & $\mathrm{CrI}$ & $0.20( \pm 0.09)$ & 0.69 & \\
\hline \multirow[t]{2}{*}{4374.94} & 4375.93 & FeI & $12.19( \pm 0.84)$ & 2.43 & \\
\hline & 4384.98 & $\mathrm{CrI}$ & & & $\mathrm{p}$ \\
\hline 4426.23 & 4427.31 & FeI & $0.47( \pm 0.07)$ & 0.58 & \\
\hline 4460.53 & 4461.65 & FeI & $0.32( \pm 0.11)$ & 0.93 & \\
\hline 4495.73 & 4496.84 & $\mathrm{CrI}$ & $0.11( \pm 0.07)$ & 0.60 & \\
\hline 4544.72 & 4545.94 & $\mathrm{CrI}$ & $0.25( \pm 0.15)$ & 1.2: & \\
\hline 4552.95 & 4554.12 & BaII & $0.23( \pm 0.07)$ & 0.59 & \\
\hline 4569.95 & 4571.10 & MgI & $4.03( \pm 0.12)$ & 0.95 & \\
\hline 4578.87 & 4580.04 & $\mathrm{CrI}$ & $0.16( \pm 0.08)$ & 0.67 & \\
\hline 4599.62 & 4600.74 & $\mathrm{CrI}$ & $0.44( \pm 0.16)$ & 1.29 & \\
\hline 4606.15 & 4607.33 & SrI & $8.85( \pm 0.48)$ & 1.33 & \\
\hline 4612.29 & 4613.36 & $\mathrm{CrI}$ & $0.20( \pm 0.12)$ & 0.92 & \\
\hline 4614.98 & 4616.12 & $\mathrm{CrI}$ & $0.39( \pm 0.12)$ & 0.92 & \\
\hline 4624.98 & 4626.17 & $\mathrm{CrI}$ & $0.65( \pm 0.13)$ & 1.04 & \\
\hline 4644.99 & 4646.15 & $\mathrm{CrI}$ & $0.86( \pm 0.12)$ & 0.98 & \\
\hline 4650.18 & 4651.28 & $\mathrm{CrI}$ & $0.13( \pm 0.06)$ & 0.50 & $\mathrm{~b}, \mathrm{c}$ \\
\hline 4650.98 & 4652.15 & $\mathrm{CrI}$ & $0.25( \pm 0.07)$ & 0.56 & $\mathrm{~b}, \mathrm{c}$ \\
\hline 4932.87 & 4934.08 & BaII & $0.33( \pm 0.06)$ & 0.59 & \\
\hline \multirow[t]{3}{*}{5109.14} & 5110.41 & FeI & $28.39( \pm 1.12)$ & 1.48 & $\mathrm{~b}$ \\
\hline & 5166.28 & FeI & & & $\mathrm{B}, \mathrm{u}$ \\
\hline & 5168.90 & FeI & & & $\mathrm{B}, \mathrm{u}$ \\
\hline 5203.07 & 5204.51 & $\mathrm{CrI}$ & $13.93( \pm 0.20)$ & 1.29: & $\mathrm{b}$ \\
\hline 5204.68 & 5206.04 & $\mathrm{CrI}$ & $16.17( \pm 0.19)$ & 1.22: & $\mathrm{b}$ \\
\hline 5207.04 & 5208.42 & $\mathrm{CrI}$ & $24.17( \pm 0.21)$ & 1.37: & $\mathrm{b}$ \\
\hline 5246.23 & 5247.57 & $\mathrm{CrI}$ & $1.15( \pm 0.14)$ & 0.91 & \\
\hline 5262.86 & 5264.15 & $\mathrm{CrI}$ & $2.46( \pm 0.12)$ & 0.79 & $\mathrm{~b}, \mathrm{c}$ \\
\hline 5264.49 & 5265.72 & $\mathrm{CrI}$ & $0.30( \pm 0.05)$ & 0.3: & $\mathrm{b}, \mathrm{c}$ \\
\hline 5268.27 & 5269.54 & FeI & $1.05( \pm 0.10)$ & 0.61 & $\mathrm{c}$ \\
\hline 5295.34 & 5296.69 & $\mathrm{CrI}$ & $2.53( \pm 0.15)$ & 0.97 & $\mathrm{~b}$ \\
\hline 5296.94 & 5298.28 & $\mathrm{CrI}$ & $2.31( \pm 0.12)$ & 0.77 & $\mathrm{~b}$ \\
\hline 5299.18 & 5300.74 & $\mathrm{CrI}$ & $0.73( \pm 0.26)$ & 1.5: & $b, f$ \\
\hline
\end{tabular}

Notes. The flux is listed in $10^{-16} \mathrm{erg} \mathrm{cm}^{-2} \mathrm{~s}^{-1}$, the wavelengths and FWHM in $\AA$. 
Table 2. continued.

\begin{tabular}{lllrll}
\hline \hline$\lambda_{\text {obs }}$ & $\lambda_{\text {lab }}$ & Identification & \multicolumn{1}{l}{ Flux } & $F W H M$ & Notes \\
\hline 5326.72 & 5328.04 & FeI & $0.99( \pm 0.24)$ & $1.4:$ & $\mathrm{s}$ \\
5344.46 & 5345.80 & CrI & $4.54( \pm 0.16)$ & 0.93 & $\mathrm{~b}$ \\
5346.94 & 5348.31 & CrI & $2.93( \pm 0.18)$ & $1.0:$ & $\mathrm{b}$ \\
& 5394.68 & MnI & $6.23( \pm 0.22)$ & 0.82 & $\mathrm{p}, \mathrm{B}, \mathrm{u}$ \\
5408.5 & 5409.77 & $\mathrm{CrI}$ & $0.58( \pm 0.14)$ & 0.84 & $\mathrm{f}, \mathrm{c}$ \\
5431.17 & 5432.55 & $\mathrm{MnI}$ & $0.96( \pm 0.22)$ & $1.4:$ & \\
5534.20 & 5535.48 & $\mathrm{BaI}$ & $0.08( \pm 0.05)$ & $0.3:$ & $\mathrm{f}, \mathrm{c}$ \\
5666.76 & 5668.28 & $\mathrm{MnI}$ & $0.21( \pm 0.10)$ & 0.65 & $\mathrm{f}, \mathrm{c}$ \\
5688.96 & 5690.43 & $\mathrm{MnI}$ & $0.43( \pm 0.26)$ & $1.6:$ & \\
5727.10 & 5728.57 & $\mathrm{MnI}$ & $0.99( \pm 0.19)$ & 1.27 & $\mathrm{c}$ \\
5861.20 & 5862.69 & $\mathrm{MnI}$ & $252.10( \pm 0.60)$ & $4.0: \mathrm{E}, \mathrm{b}$ & \\
5889.2 & 5889.95 & $\mathrm{NaI}$ & $183.35( \pm 0.40)$ & $2.8: \mathrm{E}, \mathrm{b}$ & \\
5894.6 & 5895.92 & $\mathrm{NaI}$ & $0.24( \pm 0.06)$ & $0.4:$ & $\mathrm{f}, \mathrm{b}$ \\
6279.10 & 6280.62 & FeI & $0.41( \pm 0.18)$ & $1.3:$ & $\mathrm{s}$ \\
6328.26 & 6330.09 & $\mathrm{CrI}$ & $49.19( \pm 0.17)$ & 1.12 & $\mathrm{~B}$ \\
6571.10 & 6572.78 & $\mathrm{CaI}$ & $1358.74( \pm 2.26)$ & 3.34 & $\mathrm{E}, \mathrm{b}$ \\
7663. & 7664.91 & $\mathrm{KI}$ & $1291.33( \pm 2.19)$ & 3.23 & $\mathrm{E}, \mathrm{b}$ \\
7697. & 7698.97 & KI & $37.01( \pm 2.10)$ & 2.11 & $\mathrm{~b}$ \\
7798.52 & 7800.27 & RbI & $6.15( \pm 0.49)$ & 1.55 & $\mathrm{~B}$ \\
7946.14 & 7947.60 & RbI & & & \\
\hline
\end{tabular}


Table 3. Molecular bands of the spectrum of V4332 Sgr.

\begin{tabular}{|c|c|c|c|}
\hline$\lambda_{\mathrm{lab}}$ & Identification & Flux & Notes \\
\hline 4470.5 & $\mathrm{AlO} \mathrm{B}^{2} \Sigma^{+}-\mathrm{X}^{2} \Sigma^{+}(2,0)$ & $0.38( \pm 0.06)$ & \\
\hline 4494.0 & $\mathrm{AlO} \mathrm{B}^{2} \Sigma^{+}-\mathrm{X}^{2} \Sigma^{+}(3,1)$ & $0.28( \pm 0.05)$ & $\mathrm{f}$ \\
\hline 4516.4 & $\mathrm{AlO} \mathrm{B}^{2} \Sigma^{+}-\mathrm{X}^{2} \Sigma^{+}(4,2)$ & $0.29( \pm 0.08)$ & $\mathrm{f}$ \\
\hline 4648.2 & $\mathrm{AlO} \mathrm{B}^{2} \Sigma^{+}-\mathrm{X}^{2} \Sigma^{+}(1,0)$ & $9.07( \pm 0.61)$ & $\mathrm{b}$ \\
\hline 4672.0 & $\mathrm{AlO} \mathrm{B}^{2} \Sigma^{+}-\mathrm{X}^{2} \Sigma^{+}(2,1)$ & $6.43( \pm 0.55)$ & \\
\hline 4694.6 & $\mathrm{AlO} \mathrm{B}^{2} \Sigma^{+}-\mathrm{X}^{2} \Sigma^{+}(3,2)$ & $2.26( \pm 0.60)$ & \\
\hline 4715.5 & $\mathrm{AlO} \mathrm{B}^{2} \Sigma^{+}-\mathrm{X}^{2} \Sigma^{+}(4,3)$ & $0.62( \pm 0.42)$ & $\mathrm{f}$ \\
\hline 4735.8 & $\mathrm{AlO} \mathrm{B}^{2} \Sigma^{+}-\mathrm{X}^{2} \Sigma^{+}(5,4)$ & & $\mathrm{p}$ \\
\hline 4760.9 & $\mathrm{TiO} \alpha(2,0) \mathrm{R}_{2}$ & $0.41( \pm 0.09)$ & $\mathrm{c}$ \\
\hline 4804.3 & $\mathrm{TiO} \alpha(3,1) \quad \mathrm{R}_{2}$ & & $\mathrm{p}$ \\
\hline 4842.3 & $\mathrm{AlO} \mathrm{B}^{2} \Sigma^{+}-\mathrm{X}^{2} \Sigma^{+}(0,0)$ & $43.52( \pm 0.83)$ & \\
\hline 4866.4 & $\mathrm{AlO} \mathrm{B}^{2} \Sigma^{+}-\mathrm{X}^{2} \Sigma^{+}(1,1)$ & $16.26( \pm 0.67)$ & \\
\hline 4889.0 & $\mathrm{AlO} \mathrm{B}^{2} \Sigma^{+}-\mathrm{X}^{2} \Sigma^{+}(2,2)$ & $3.63( \pm 0.89)$ & $\mathrm{c}$ \\
\hline 4954.6 & $\mathrm{TiO} \alpha(1,0)$ & $5.02( \pm 0.51)$ & $\mathrm{c}$ \\
\hline 4999.1 & $\mathrm{TiO} \alpha(2,1)$ & $2.82( \pm 0.74)$ & $\mathrm{c}$ \\
\hline 5010.5 & VO $C^{4} \Sigma^{-}-X^{4} \Sigma^{-}(3,0)$ & $2.32( \pm 0.29)$ & $\mathrm{s}, \mathrm{c}$ \\
\hline 5079.4 & $\mathrm{AlO} \mathrm{B}^{2} \Sigma^{+}-\mathrm{X}^{2} \Sigma^{+}(0,1)$ & $31.41( \pm 0.90)$ & \\
\hline 5102.1 & $\mathrm{AlO} \mathrm{B}^{2} \Sigma^{+}-\mathrm{X}^{2} \Sigma^{+}(1,2)$ & $17.71( \pm 0.92)$ & $\mathrm{b}$ \\
\hline 5123.3 & $\mathrm{AlO} \mathrm{B}^{2} \Sigma^{+}-\mathrm{X}^{2} \Sigma^{+}(2,3)$ & $7.83( \pm 0.94)$ & \\
\hline 5142.9 & $\mathrm{AlO} \mathrm{B}^{2} \Sigma^{+}-\mathrm{X}^{2} \Sigma^{+}(3,4)$ & $1.94( \pm 0.57)$ & \\
\hline 5161.0 & $\mathrm{AlO} \mathrm{B}^{2} \Sigma^{+}-\mathrm{X}^{2} \Sigma^{+}(4,5)$ & & $\mathrm{p}$ \\
\hline 5166.7 & $\mathrm{TiO} \alpha(0,0)$ & $14.46( \pm 0.52)$ & 古 \\
\hline 5166.3 & FeI & & |B \\
\hline 5168.9 & FeI & & 」B \\
\hline 5228.2 & VO $C^{4} \Sigma^{-}-X^{4} \Sigma^{-}(2,0)$ & $1.15( \pm 0.32)$ & $\mathrm{f}, \mathrm{c}$ \\
\hline 5275.8 & $\operatorname{VO} C^{4} \Sigma^{-}-X^{4} \Sigma^{-}(3,1)$ & & $\mathrm{p}$ \\
\hline 5336.5 & $\mathrm{AlO} \mathrm{B}^{2} \Sigma^{+}-\mathrm{X}^{2} \Sigma^{+}(0,2)$ & $2.07( \pm 0.41)$ & $\mathrm{b}$ \\
\hline 5357.6 & $\mathrm{AlO} \mathrm{B}^{2} \Sigma^{+}-\mathrm{X}^{2} \Sigma^{+}(1,3)$ & $2.43( \pm 0.88)$ & $\mathrm{c}$ \\
\hline 5376.8 & $\mathrm{AlO} \mathrm{B}{ }^{2} \Sigma^{+}-\mathrm{X}^{2} \Sigma^{+}(2,4)$ & $1.61( \pm 1.13)$ & $\mathrm{f}, \mathrm{c}$ \\
\hline 5448.2 & $\mathrm{TiO} \alpha(0,1)$ & $10.13( \pm 1.42)$ & \\
\hline 5469.3 & VO $C^{4} \Sigma^{-}-X^{4} \Sigma^{-}(1,0)$ & $9.84( \pm 1.80)$ & \\
\hline 5496.4 & $\mathrm{TiO} \alpha(1,2)$ & $7.33( \pm 1.40)$ & \\
\hline 5517.3 & $\operatorname{VOC} C^{4} \Sigma^{-}-X^{4} \Sigma^{-}(2,1)$ & $1.85( \pm 0.92)$ & $\mathrm{f}, \mathrm{c}$ \\
\hline 5564.3 & $\mathrm{CrO} \mathrm{B}^{5} \Pi_{-1}-\mathrm{X}^{5} \Pi_{-1}(2,0)$ & $3.79( \pm 0.97)$ & ךB \\
\hline 5565.9 & $\mathrm{CrO} \mathrm{B}^{5} \Pi_{0}-\mathrm{X}^{5} \Pi_{0}(2,0)$ & & B \\
\hline 5567.7 & $\mathrm{CrO} \mathrm{B}{ }^{5} \Pi_{1}-\mathrm{X}^{5} \Pi_{1}(2,0)$ & & B \\
\hline 5570.2 & $\mathrm{CrO} \mathrm{B}{ }^{5} \Pi_{2}-\mathrm{X}^{5} \Pi_{2}(2,0)$ & & B \\
\hline 5576.4 & $\mathrm{CrO} \mathrm{B}{ }^{5} \Pi_{3}-\mathrm{X}^{5} \Pi_{3}(2,0)$ & & \rfloor $\mathrm{B}$ \\
\hline 5736.7 & VO $C^{4} \Sigma^{-}-X^{4} \Sigma^{-}(0,0)$ & $10.47( \pm 1.14)$ & $\mathrm{c}$ \\
\hline 5847.6 & $\mathrm{TiO} \gamma^{\prime}(1,0) \mathrm{F}_{1}-\mathrm{F}_{1}$ & $7.22( \pm 0.65)$ & $\mathrm{c}$ \\
\hline 5872.7 & $\mathrm{TiO} \gamma^{\prime}(1,0) \mathrm{F}_{2}-\mathrm{F}_{2}$ & $5.14( \pm 0.87)$ & \\
\hline 5898.9 & $\mathrm{TiO} \gamma^{\prime}(1,0) \mathrm{Q}_{3}, \mathrm{P}_{3}$ & & $\mathrm{p}, \mathrm{B}$ \\
\hline 5821.2 & $\mathrm{TiO} \gamma^{\prime}(2,1) \mathrm{F}_{2}-\mathrm{F}_{2}$ & $1.50( \pm 0.43)$ & \\
\hline 5947.7 & $\mathrm{TiO} \gamma^{\prime}(2,1) \mathrm{R}_{3}$ & $0.90( \pm 0.35)$ & \\
\hline 5954.4 & $\mathrm{TiO} \gamma^{\prime}(2,1) \mathrm{Q}_{3}, \mathrm{P}_{3}$ & $1.74( \pm 0.37)$ & \\
\hline 5972.2 & YO $A^{2} \Pi_{3 / 2}-X^{2} \Sigma^{+}(0,0)$ & $5.24( \pm 0.55)$ & \\
\hline 6036.2 & $\mathrm{ScO} \mathrm{A}^{2} \Pi_{3 / 2}-\mathrm{X}^{2} \Sigma^{+}(0,0)$ & $51.23( \pm 2.02)$ & \\
\hline 6051.8 & $\mathrm{CrO} \mathrm{B}^{5} \Pi_{-1}-\mathrm{X}^{5} \Pi_{-1}(0,0)$ & $21.66( \pm 1.63)$ & \rceil $\mathrm{B}$ \\
\hline 6053.3 & $\mathrm{CrO} \mathrm{B}^{5} \Pi_{0}-\mathrm{X}^{5} \Pi_{0}(0,0)$ & & B \\
\hline 6054.8 & $\mathrm{CrO} \mathrm{B}^{5} \Pi_{1}-\mathrm{X}^{5} \Pi_{1}(0,0)$ & & $\mathrm{B}$ \\
\hline 6058.5 & $\mathrm{CrO} \mathrm{B}^{5} \Pi_{2}-\mathrm{X}^{5} \Pi_{2}(0,0)$ & & $\mathrm{B}$ \\
\hline 6063.5 & $\mathrm{CrO} \mathrm{B}{ }^{5} \Pi_{3}-\mathrm{X}^{5} \Pi_{3}(0,0)$ & & B \\
\hline 6064.3 & $\mathrm{ScO} \mathrm{A}^{2} \Pi_{1 / 2}-\mathrm{X}^{2} \Sigma^{+}(0,0){ }^{R} R_{1 G}$ & & $\mathrm{~J}, \mathrm{p}$ \\
\hline
\end{tabular}

Notes. The flux is listed in $10^{-16} \mathrm{erg} \mathrm{cm}^{-2} \mathrm{~s}^{-1}$, the wavelengths in $\AA$. 
Table 3. continued.

\begin{tabular}{|c|c|c|c|}
\hline$\lambda_{\mathrm{lab}}$ & Identification & Flux & Notes \\
\hline 6072.6 & $\mathrm{ScO}^{2} \Pi_{3 / 2}-\mathrm{X}^{2} \Sigma^{+}(1,1)$ & $2.44( \pm 0.22)$ & $\mathrm{b}$ \\
\hline 6079.3 & $\mathrm{ScO} \mathrm{A}^{2} \Pi_{1 / 2}-\mathrm{X}^{2} \Sigma^{+}(0,0)$ & $74.26( \pm 2.49)$ & \rceil $\mathrm{B}$ \\
\hline 6086.4 & VO $C^{4} \Sigma^{-}-X^{4} \Sigma^{-}(0,1)$ & & $\mathrm{B}$ \\
\hline 6116.0 & $\operatorname{ScO} A^{2} \Pi_{1 / 2}-X^{2} \Sigma^{+}(1,1)$ & $3.12( \pm 0.60)$ & \\
\hline 6132.1 & YO A ${ }^{2} \Pi_{1 / 2}-X^{2} \Sigma^{+}(0,0)$ & $11.16( \pm 1.01)$ & \rceil $\mathrm{B}$ \\
\hline 6138.8 & VO $C^{4} \Sigma^{-}-X^{4} \Sigma^{-}(1,2)$ & & JB \\
\hline 6148.7 & $\mathrm{TiO} \gamma^{\prime}(0,0) \mathrm{R}_{21}$ & $1.95( \pm 0.28)$ & $\mathrm{b}$ \\
\hline 6158.5 & $\mathrm{TiO} \gamma^{\prime}(0,0) \mathrm{F}_{1}-\mathrm{F}_{1}$ & $63.61( \pm 1.86)$ & \\
\hline 6183.2 & $\mathrm{TiO} \gamma^{\prime}(0,0) \mathrm{Q}_{32}$ & $2.37( \pm 0.19)$ & $\mathrm{b}$ \\
\hline 6186.3 & $\mathrm{TiO} \gamma^{\prime}(0,0) \mathrm{F}_{2}-\mathrm{F}_{2}$ & $37.64( \pm 1.44)$ & \\
\hline 6210.8 & $\mathrm{TiO} \gamma^{\prime}(1,1) \mathrm{R}_{1}$ & $3.27( \pm 0.22)$ & B \\
\hline 6214.9 & $\mathrm{TiO} \gamma^{\prime}(0,0) \mathrm{F}_{3}-\mathrm{F}_{3}$ & $45.47( \pm 1.64)$ & $\mathrm{b}$ \\
\hline 6239.0 & $\mathrm{TiO} \gamma^{\prime}(1,1) \mathrm{R}_{2}$ & $0.98( \pm 0.08)$ & \\
\hline 6242.2 & $\mathrm{TiO} \gamma^{\prime}(1,1) \mathrm{Q}_{2}, \mathrm{P}_{2}$ & $5.75( \pm 0.55)$ & \\
\hline 6268.9 & $\mathrm{TiO} \gamma^{\prime}(1,1) \mathrm{F}_{3}-\mathrm{F}_{3}$ & $7.30( \pm 1.07)$ & $\mathrm{b}$ \\
\hline 6321.2 & $\mathrm{TiO} \gamma(2,0) \mathrm{R}_{2}$ & $0.28( \pm 0.07)$ & $\mathrm{f}$ \\
\hline 6351.3 & $\mathrm{TiO} \gamma^{\prime}(2,2) \mathrm{Q}_{3}$ & $1.33( \pm 0.46)$ & $\mathrm{c}, \mathrm{f}$ \\
\hline 6394.2 & $\mathrm{CrO} \mathrm{B}^{5} \Pi_{-1}-\mathrm{X} \Pi_{-1}(0,1)$ & $33.37( \pm 2.00)$ & \rceil $\mathrm{B}$ \\
\hline 6396.2 & $\mathrm{CrO} \mathrm{B}^{5} \Pi_{0}-\mathrm{X} \Pi_{0}(0,1)$ & & B \\
\hline 6397.8 & $\mathrm{CrO} \mathrm{B}^{5} \Pi_{1}-\mathrm{X} \Pi_{1}(0,1)$ & & $\mathrm{B}$ \\
\hline 6401.4 & $\mathrm{CrO} \mathrm{B}^{5} \Pi_{2}-\mathrm{X} \Pi_{2}(0,1)$ & & $\mathrm{B}$ \\
\hline 6407.7 & $\mathrm{CrO} \mathrm{B}^{5} \Pi_{3}-\mathrm{X \Pi}_{3}(0,1)$ & & $\mathrm{B}$ \\
\hline 6451.7 & $\mathrm{CrO} \mathrm{B}^{5} \Pi_{-1}-\mathrm{X} \Pi_{-1}(1,2)$ & $14.65( \pm 1.92)$ & B \\
\hline 6451.9 & $\mathrm{CrO} \mathrm{B}^{5} \Pi_{0}-\mathrm{X}^{5} \Pi_{0}(1,2)$ & & B \\
\hline 6455.2 & $\mathrm{CrO} \mathrm{B}^{5} \Pi_{1}-\mathrm{X}^{5} \Pi_{1}(1,2)$ & & $\mathrm{B}$ \\
\hline 6459.5 & $\mathrm{CrO} \mathrm{B}^{5} \Pi_{2}-\mathrm{X}^{5} \Pi_{2}(1,2)$ & & B \\
\hline 6465.4 & $\mathrm{CrO} \mathrm{B}^{5} \Pi_{3}-\mathrm{X}^{5} \Pi_{3}(1,2)$ & & $\mathrm{B}$ \\
\hline 6477.8 & VO $C^{4} \Sigma^{-}-X^{4} \Sigma^{-}(0,2)$ & $5.46( \pm 1.30)$ & $\mathrm{b}$ \\
\hline 6533.3 & VO $C^{4} \Sigma^{-}-X^{4} \Sigma^{-}(1,3)$ & $11.48( \pm 2.08)$ & $\mathrm{b}$ \\
\hline 6562.6 & $\mathrm{TiO} \gamma^{\prime}(0,1) \mathrm{F}_{1}-\mathrm{F}_{1}$ & $24.37( \pm 1.02)$ & $\mathrm{B}$ \\
\hline 6594.0 & $\mathrm{TiO} \gamma^{\prime}(0,1) \mathrm{R}_{2}$ & $11.84( \pm 1.22)$ & \rceil $\mathrm{B}$ \\
\hline 6597.9 & $\mathrm{TiO} \gamma^{\prime}(0,1) \mathrm{Q}_{2}, \mathrm{P}_{2}$ & & $\mathrm{~B}$ \\
\hline 6618.0 & $\mathrm{TiO} \gamma^{\prime}(1,2) \mathrm{F}_{1}-\mathrm{F}_{1}$ & $8.18( \pm 1.00)$ & c \\
\hline 6635.4 & $\mathrm{TiO} \gamma^{\prime}(0,1) \mathrm{Q}_{3}, \mathrm{P}_{3}$ & $5.70( \pm 0.51)$ & \\
\hline 6651.3 & $\mathrm{TiO} \gamma(1,0) \mathrm{F}_{3}-\mathrm{F}_{3}$ & $12.07( \pm 0.66)$ & $\mathrm{c},\rceil \mathrm{B}$ \\
\hline 6649.8 & $\mathrm{TiO} \gamma^{\prime}(1,2) \mathrm{F}_{2}-\mathrm{F}_{2}$ & & $\mathrm{~B}$ \\
\hline 6680.8 & $\mathrm{TiO} \gamma(1,0) \mathrm{F}_{2}-\mathrm{F}_{2}$ & $12.58( \pm 1.50)$ & \rceil $\mathrm{B}$ \\
\hline 6681.8 & $\mathrm{TiO} \gamma^{\prime}(1,2) \mathrm{F}_{3}-\mathrm{F}_{3}$ & & JB \\
\hline 6714.5 & $\mathrm{TiO} \gamma(1,0) \mathrm{F}_{1}-\mathrm{F}_{1}$ & $25.94( \pm 1.90)$ & \rceil $\mathrm{B}$ \\
\hline 6717.6 & $\mathrm{TiO} \gamma(2,1) \mathrm{F}_{3}-\mathrm{F}_{3}$ & & JB \\
\hline 6747.6 & $\mathrm{TiO} \gamma(2,1) \mathrm{F}_{2}-\mathrm{F}_{2}$ & $9.64( \pm 1.54)$ & \\
\hline 6772.3 & $\mathrm{CrO}^{5} \Pi_{-1}-\mathrm{X}^{5} \Pi_{-1}(0,2)$ & $24.66( \pm 1.89)$ & \rceil $\mathrm{B}$ \\
\hline 6774.2 & $\mathrm{CrO} \mathrm{B}^{5} \Pi_{0}-\mathrm{X}^{5} \Pi_{0}(0,2)$ & & B \\
\hline 6775.9 & $\mathrm{CrO} \mathrm{B}^{5} \Pi_{1}-\mathrm{X}^{5} \Pi_{1}(0,2)$ & & B \\
\hline 6779.6 & $\mathrm{CrO} \mathrm{B}^{5} \Pi_{2}-\mathrm{X}^{5} \Pi_{2}(0,2)$ & & B \\
\hline 6781.8 & $\mathrm{TiO} \gamma(2,1) \mathrm{F}_{1}-\mathrm{F}_{1}$ & & B \\
\hline 6785.7 & $\mathrm{CrO} \mathrm{B}^{5} \Pi_{3}-\mathrm{X}^{5} \Pi_{3}(0-2)$ & & JB \\
\hline 6815.1 & $\mathrm{TiO} \gamma(3,2) \mathrm{F}_{2}-\mathrm{F}_{2}$ & $0.51( \pm 0.18)$ & $\mathrm{f}$ \\
\hline 6830.7 & $\mathrm{TiO} \mathrm{b}^{1} \Pi-\mathrm{X}^{3} \Pi_{1}(0,0) \mathrm{R}_{12}$ & $18.24( \pm 2.45)$ & \rceil $\mathrm{B}$ \\
\hline 6836.5 & $\mathrm{CrO}^{5} \Pi_{-1}-\mathrm{X}^{5} \Pi_{-1}(1,3)$ & & B \\
\hline 6836.5 & $\mathrm{TiO} b^{1} \Pi-X^{3} \Pi_{1}(0,0) Q_{12}$ & & B \\
\hline 6836.6 & $\mathrm{CrO} \mathrm{B}^{5} \Pi_{0}-\mathrm{X}^{5} \Pi_{0}(1,3)$ & & B \\
\hline 6839.9 & $\mathrm{CrO} \mathrm{B}^{5} \Pi_{1}-\mathrm{X}^{5} \Pi_{1}(1,3)$ & & B \\
\hline 6844.5 & $\mathrm{CrO} \mathrm{B}^{5} \Pi_{2}-\mathrm{X}^{5} \Pi_{2}(1,3)$ & & B \\
\hline 6850.9 & $\mathrm{CrO} \mathrm{B}^{5} \Pi_{3}-\mathrm{X}^{5} \Pi_{3}(1,3)$ & & JB \\
\hline 6919.0 & VO $C^{4} \Sigma^{-}-X^{4} \Sigma^{-}(0,3)$ & $1.51( \pm 0.43)$ & $\mathrm{f}, \mathrm{E}$ \\
\hline 6976.2 & VO $C^{4} \Sigma^{-}-X^{4} \Sigma^{-}(1,4)$ & $1.05( \pm 0.42)$ & $\mathrm{f}, \mathrm{c}$ \\
\hline
\end{tabular}


Table 3. continued.

\begin{tabular}{|c|c|c|c|}
\hline$\lambda_{\text {lab }}$ & Identification & Flux & Notes \\
\hline 7054.2 & $\mathrm{TiO} \gamma(0,0) \mathrm{F}_{3}-\mathrm{F}_{3}$ & $119.27( \pm 3.68)$ & \\
\hline 7087.6 & $\mathrm{TiO} \gamma(0,0) \mathrm{F}_{2}-\mathrm{F}_{2}$ & $118.40( \pm 3.85)$ & \\
\hline 7125.5 & $\mathrm{TiO} \gamma(0,0) \mathrm{F}_{1}-\mathrm{F}_{1}$ & $184.10( \pm 3.67)$ & \rceil $\mathrm{B}$ \\
\hline 7124.9 & $\mathrm{TiO} \gamma(1,1) \mathrm{F}_{3}-\mathrm{F}_{3}$ & & \rfloor $\mathrm{B}$ \\
\hline 7158.8 & $\mathrm{TiO} \gamma(1,1) \mathrm{F}_{2}-\mathrm{F}_{2}$ & $26.39( \pm 3.40)$ & $\mathrm{E}$ \\
\hline 7197.4 & $\mathrm{TiO} \gamma(1,1) \mathrm{F}_{1}-\mathrm{F}_{1}$ & $33.24( \pm 2.42)$ & ךB E, s \\
\hline 7196.4 & $\mathrm{TiO} \gamma(2,2) \mathrm{F}_{3}-\mathrm{F}_{3}$ & & \rfloor $\mathrm{B}$ \\
\hline 7270.1 & $\mathrm{TiO} \gamma(2,2) \mathrm{F}_{1}-\mathrm{F}_{1}$ & $0.73( \pm 0.26)$ & $\mathrm{c}, \mathrm{f}$ \\
\hline 7343 & VO B ${ }^{4} \Pi_{5 / 2}-X^{4} \Sigma^{-}(1,0)$ & $14.38( \pm 2.06)$ & $\mathrm{c}$ \\
\hline 7378 & VO $B^{4} \Pi_{3 / 2}-X^{4} \Sigma^{-}(1,0)$ & $17.74( \pm 3.61)$ & $\mathrm{c}$ \\
\hline 7408 & VO $B^{4} \Pi_{1 / 2}-X^{4} \Sigma^{-}(1,0)$ & $17.23( \pm 4.42)$ & $\mathrm{c}$ \\
\hline 7454 & VO $\mathrm{B}^{4} \Pi_{-1 / 2}-\mathrm{X}^{4} \Sigma^{-}(1,0)$ & $21.77( \pm 3.94)$ & $\mathrm{c}$ \\
\hline 7589.3 & $\mathrm{TiO} \gamma \mathrm{F}_{3}-\mathrm{F}_{3}(0,1)$ & $>14.01( \pm 0.75)$ & \\
\hline 7627.7 & $\mathrm{TiO} \gamma \mathrm{F}_{2}-\mathrm{F}_{2}(0,1)$ & & $\mathrm{p}, \mathrm{u}, \mathrm{s}$ \\
\hline 7665.8 & $\mathrm{TiO} \gamma \mathrm{F}_{3}-\mathrm{F}_{3}(1,2)$ & & $\mathrm{B}, \mathrm{u}$ \\
\hline 7671.6 & $\mathrm{TiO} \gamma \mathrm{F}_{1}-\mathrm{F}_{1}(0,1)$ & & $\mathrm{p}, \mathrm{B}, \mathrm{u}$ \\
\hline 7704.9 & $\mathrm{TiO} \gamma \mathrm{F}_{2}-\mathrm{F}_{2}(1,2)$ & & $\mathrm{p}, \mathrm{B}, \mathrm{u}$ \\
\hline 7743.0 & $\mathrm{TiO} \gamma \mathrm{F}_{3}-\mathrm{F}_{3}(2,3)$ & & $\mathrm{p}, \mathrm{B}$ \\
\hline 7749.5 & $\mathrm{TiO} \gamma \mathrm{F}_{1}-\mathrm{F}_{1}(1,2)$ & $27.48( \pm 3.71$ & \\
\hline 7782.8 & $\mathrm{TiO} \gamma \mathrm{F}_{2}-\mathrm{F}_{2}(2,3)$ & $4.77( \pm 1.64)$ & $\mathrm{b}$ \\
\hline 7828.1 & $\mathrm{TiO} \gamma \mathrm{F}_{1}-\mathrm{F}_{1}(2,3)$ & $5.69( \pm 1.85)$ & $\mathrm{f}$ \\
\hline 7867.1 & VO $\mathrm{B}^{4} \Pi_{5 / 2}-\mathrm{X}^{4} \Sigma^{-}(0,0)$ & $87.16( \pm 7.38)$ & $\mathrm{b}$ \\
\hline 7910.4 & VO $B^{4} \Pi_{3 / 2}-X^{4} \Sigma^{-}(0,0)$ & $87.96( \pm 7.15)$ & $\mathrm{b}$ \\
\hline 7941.9 & VO $B^{4} \Pi_{1 / 2}-X^{4} \Sigma^{-}(0,0)$ & 100.888: $( \pm 6.321)$ & $\mathrm{b}$ \\
\hline 7982.6 & VO $B^{4} \Pi_{-1 / 2}-X^{4} \Sigma^{-}(0,0)$ & $158.93( \pm 13.30)$ & $\mathrm{b}$ \\
\hline 8030.3 & VO $B^{4} \Pi_{-1 / 2}-X^{4} \Sigma^{-}(1,1)$ & $19.69( \pm 4.72)$ & \\
\hline 8433.2 & $\mathrm{TiO} \in(0,0) \mathrm{R}_{1}$ & $76.36( \pm 6.87)$ & \rceil $\mathrm{B}$ \\
\hline 8442.3 & $\mathrm{TiO} \epsilon(0,0) \mathrm{R}_{2}, \mathrm{Q}_{1}, \mathrm{P}_{1}$ & & B \\
\hline 8451.8 & $\mathrm{TiO} \epsilon(0,0) \mathrm{R}_{3}, \mathrm{Q}_{2}, \mathrm{P}_{2}$ & & B \\
\hline 8462.7 & $\mathrm{TiO} \epsilon(0,0) \mathrm{Q}_{3}, \mathrm{P}_{3}$ & & JB \\
\hline 8682.6 & VO $B^{4} \Pi_{-1 / 2}-X^{4} \Sigma^{-}(0,1)$ & $33.45( \pm 3.67)$ & $\mathrm{c}$ \\
\hline 8736.7 & VO $B^{4} \Pi_{-1 / 2}-X^{4} \Sigma^{-}(1,2) R_{1}$ & $2.62( \pm 0.66)$ & $\mathrm{f}$ \\
\hline 8760.0 & VO $B^{4} \Pi_{-1 / 2}-X^{4} \Sigma^{-}(1,2) P_{1}$ & $7.58( \pm 1.70)$ & $\mathrm{c}$ \\
\hline
\end{tabular}

Table 4. Meaning of symbols used in Tables 2 and 3.

\begin{tabular}{rll}
\hline \hline JB & - measurements refer to the whole blend \\
$\mathrm{c}$ & - & uncertain continuum level \\
$\mathrm{f}$ & - & faint feature \\
$\mathrm{s}$ & - & shape-fitting was problematical \\
$\mathrm{b}$ & - & blended features \\
$\mathrm{B}$ & - & very strong blending \\
$\mathrm{E}$ & - & corrected for interstellar and/or telluric absorption \\
$:$ & - & uncertain \\
$>$ & - & lower limit \\
$\mathrm{p}$ & - & present or probably present, but unmeasurable \\
$\mathrm{u}$ & - & unmeasurable (cannot be deconvolved) \\
\hline
\end{tabular}

